\title{
Attenuating vascular stenosis-induced astrogliosis preserves white matter integrity and cognitive function
}

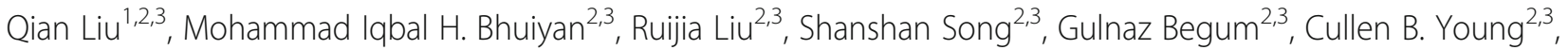
Lesley M. Foley ${ }^{4}$ Fenghua Chen ${ }^{2}$, T. Kevin Hitchens ${ }^{4,5}$, Guodong Cao ${ }^{2,6}$, Ansuman Chattopadhyay ${ }^{7}$, Li He ${ }^{1 *}$ and Dandan $\operatorname{Sun}^{2,3,6^{*}}$

\begin{abstract}
Background: Chronic cerebral hypoperfusion $(\mathrm{CCH})$ causes white matter damage and cognitive impairment, in which astrogliosis is the major pathology. However, underlying cellular mechanisms are not well defined. Activation of $\mathrm{Na}^{+} / \mathrm{H}^{+}$exchanger-1 (NHE1) in reactive astrocytes causes astrocytic hypertrophy and swelling. In this study, we examined the role of NHE1 protein in astrogliosis, white matter demyelination, and cognitive function in a murine $\mathrm{CCH}$ model with bilateral carotid artery stenosis (BCAS).

Methods: Sham, BCAS, or BCAS mice receiving vehicle or a selective NHE1 inhibitor HOE642 were monitored for changes of the regional cerebral blood flow and behavioral performance for 28 days. Ex vivo MRI-DTI was subsequently conducted to detect brain injury and demyelination. Astrogliosis and demyelination were further examined by immunofluorescence staining. Astrocytic transcriptional profiles were analyzed with bulk RNAsequencing and RT-qPCR.

Results: Chronic cerebral blood flow reduction and spatial working memory deficits were detected in the BCAS mice, along with significantly reduced mean fractional anisotropy (FA) values in the corpus callosum, external capsule, and hippocampus in MRI DTI analysis. Compared with the sham control mice, the BCAS mice displayed demyelination and axonal damage and increased $\mathrm{GFAP}^{+}$astrocytes and $\mathrm{Iba} 1^{+}$microglia. Pharmacological inhibition of NHE1 protein with its inhibitor HOE642 prevented the BCAS-induced gliosis, damage of white matter tracts and hippocampus, and significantly improved cognitive performance. Transcriptome and immunostaining analysis further revealed that NHE1 inhibition specifically attenuated pro-inflammatory pathways and NADPH oxidase activation.
\end{abstract}

Conclusion: Our study demonstrates that NHE1 protein is involved in astrogliosis with pro-inflammatory transformation induced by $\mathrm{CCH}$, and its blockade has potentials for reducing astrogliosis, demyelination, and cognitive impairment.

Keywords: Hypoperfusion, Demyelination, Gliosis, $\mathrm{Na}^{+} / \mathrm{H}^{+}$exchanger, Vascular dementia

\footnotetext{
* Correspondence: heli2003new@126.com; sund@upmc.edu

'Department of Neurology, West China Hospital, Sichuan University, Chengdu 610041, Sichuan, China

${ }^{2}$ Department of Neurology, University of Pittsburgh, Pittsburgh, Pennsylvania 15213, USA

Full list of author information is available at the end of the article
}

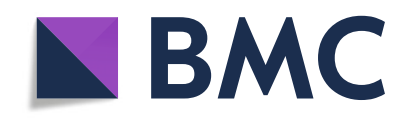

(- The Author(s). 2021 Open Access This article is licensed under a Creative Commons Attribution 4.0 International License, which permits use, sharing, adaptation, distribution and reproduction in any medium or format, as long as you give appropriate credit to the original author(s) and the source, provide a link to the Creative Commons licence, and indicate if changes were made. The images or other third party material in this article are included in the article's Creative Commons licence, unless indicated otherwise in a credit line to the material. If material is not included in the article's Creative Commons licence and your intended use is not permitted by statutory regulation or exceeds the permitted use, you will need to obtain permission directly from the copyright holder. To view a copy of this licence, visit http://creativecommons.org/licenses/by/4.0/. The Creative Commons Public Domain Dedication waiver (http://creativecommons.org/publicdomain/zero/1.0/) applies to the data made available in this article, unless otherwise stated in a credit line to the data. 


\section{Background}

Vascular contributions to cognitive impairment and dementia (VCID) have been identified as an important vascular pathologic process in the initiation and progression of vascular dementia and Alzheimer's disease (AD), which together account for approximately $60-80 \%$ of dementia worldwide $[1,2]$. Chronic cerebral hypoperfusion $(\mathrm{CCH})$ resulting from either large or small cerebral vessel diseases (such as carotid atherosclerosis or arteriosclerosis) causes subsequent white matter lesions (WMLs) and cognitive impairment and dementia [3-5]. Carotid stenosis-induced cerebral hypoperfusion is an independent risk factor for WMLs and cognitive impairment [5-7], with severe stenosis causing pronounced cognitive impairment [6,7]. Characteristic pathology of VCID includes white matter lesion, cerebral atrophy, gliosis, and endothelial damage, in part resulting from oxidative stress and neuroinflammation $[3,8]$. However, the underlying cellular mechanisms for hypoperfusion-induced WMLs and VCID are not well understood, and there is an urgent need to better understand its pathogenesis and develop therapies for the prevention and/or treatment of VCID.

Reactive glial cells, chronic inflammation, and oxidative stress are closely correlated with neurodegeneration and cognitive impairment $[9,10]$. Glial activation was detected in the early stage of AD patients, and the subsequent glia-mediated inflammatory process was suggested to lead to cognitive impairment progression [11, 12]. Serum and cerebrospinal fluid inflammatory biomarkers in older adults were significantly associated with cerebral small vessel disease and cognitive decline [13, 14]. Experimental data from a chronic hypoperfusion-induced murine VCID model showed that gliosis and a sustained inflammatory response play an important role in white matter lesion development [15]. Activation of astrocytic $\mathrm{Na}^{+} / \mathrm{H}^{+}$exchanger 1 (NHE1) causes hypertrophy and swelling of reactive astrocytes after acute brain injury $[16,17]$. In reactive astrocytes or activated microglia, NHE1 protein plays an important role in regulating intracellular $\mathrm{pH}\left(\mathrm{pH}_{\mathrm{i}}\right)$ homeostasis by extrusion of $\mathrm{H}^{+}$in exchange for $\mathrm{Na}^{+}$[18-21]. NHE1-mediated $\mathrm{H}^{+}$extrusion promotes sustained NADPH oxidase (NOX) function and pro-inflammatory responses by maintaining an alkaline $\mathrm{pH}_{\mathrm{i}}$ in microglia [19]. Moreover, increases in intracellular $\mathrm{Na}^{+}$in reactive astrocytes following NHE1 protein activation trigger a reversal of $\mathrm{Na}^{+} / \mathrm{Ca}^{2+}$ exchange and stimulation of the $\mathrm{Ca}^{2+}$-dependent signal pathways, including the release of glutamate and cytokines from astrocytes [20, 22]. In a mouse neonatal hypoxia-ischemia brain injury model, pharmacological inhibition of NHE1 by its potent inhibitor HOE642 reduced corpus callosum white matter damage and improved cognitive function [23]. These studies demonstrated that activation of glial NHE1 protein is involved in gliosis and neuroinflammation after acute ischemic or hypoxia neonatal brain injury. However, whether NHE1 protein activation plays a role in astrogliosis in chronic hypoperfusion-induced brain injury remains unknown. In this study, using a well-established murine bilateral carotid artery stenosis (BCAS) model for $\mathrm{CCH}$, we detected increased $\mathrm{GFAP}^{+}$astrocytes and $\mathrm{Iba}^{+}$microglia exhibiting NHE1 protein expression. Post-BCAS administration of the selective NHE1 inhibitor HOE642 significantly decreased astrogliosis, preserved white matter and hippocampus integrity, and improved cognitive function by preventing astrocytic ROS production and inflammatory transcriptomes. These findings revealed the potential of pharmacological blockade of NHE1 protein in reducing cerebral hypoperfusion-induced chronic brain injury and cognitive impairment.

\section{Methods \\ Materials}

Vendor and material information were included in the Supplemental data.

\section{Animals and BCAS model}

All animal studies were approved by the University of Pittsburgh Medical Center Institutional Animal Care and Use Committee, which adhere to the National Institutes of Health Guide for the Care and Use of Laboratory Animals, and reported in accordance with the Animal Research: Reporting In Vivo Experiments (ARRIVE) guidelines [24]. All efforts were made to minimize animal suffering and the number of animals used.

C57BL/6J male mice (aged 9-12 weeks, weighing 25 to $30 \mathrm{~g})$ were subjected to sham or BCAS surgery procedures. To induce BCAS, mice were anesthetized with $1.5 \%$ isoflurane in $70 \% \mathrm{~N}_{2} \mathrm{O}$ and $30 \% \mathrm{O}_{2}$ and placed in the supine position. Body temperature was maintained at $36.5 \pm 0.5{ }^{\circ} \mathrm{C}$ with a heating pad. Through a midline incision, the common carotid artery was carefully exposed and isolated from the vagus nerve and surrounding tissues. After gently lifting the carotid artery, the steel spring microcoil $(0.18-\mathrm{mm}$ internal diameter, WUXI SAMINI SPRING Co., Ltd.) was twined by rotating around the common carotid artery and placed below the carotid bifurcation. After suturing, $50 \mu \mathrm{l}$ of $0.25 \%$ bupivacaine hydrochloride was placed on the top of the incision for local infiltration anesthesia. Animals were returned to the normal cage to recover with free access to food and water.

\section{Administration of NHE1 inhibitor HOE642}

BCAS mice were randomly allocated to receive either DMSO+saline vehicle $(0.5 \%$ DMSO in saline, $n=8)$ or 
HOE642 $(0.3 \mathrm{mg} / \mathrm{kg} /$ day, $n=8)$ via intraperitoneal (i.p.) injection daily from 3-30 days after BCAS surgery. A separate group of mice $(n=9)$ was implanted with an osmotic mini-pump (Alzet, type 1004, Durect corporation, Cupertino, CA) to constantly deliver HOE642 (0.3 $\mathrm{mg} / \mathrm{kg} /$ day at a rate of $208.3 \mathrm{ng} / \mathrm{kg} / \mathrm{min}$ ) from onset to 28 days after BCAS surgery. Naïve control $(n=3)$ or sham control mice (which underwent bilateral common carotid artery isolation procedures without micro-coils placement, $n=3-5$ ) and 3 BCAS mice received no treatments.

\section{Cerebral blood flow measurement}

$\mathrm{CBF}$ in mice was measured using a two-dimensional laser speckle contrast analysis system (PeriCam PSI High Resolution with PIMSoft, Perimed, Sweden) as described before [16]. After mice were anesthetized with $1.5 \%$ isoflurane in $70 \% \mathrm{~N}_{2} \mathrm{O}$ and $30 \% \mathrm{O}_{2}$, a midline incision was made in the scalp and the exposed skull surface was cleaned with sterile normal saline. Raw speckle images of regions of interest (ROIs) covering the parietal lobe in each hemisphere were taken with a camera placed 10 $\mathrm{cm}$ above the skull. Regional CBF (rCBF) values (arbitrary perfusion units) were measured for same size areas in both parietal lobes at baseline, 5-10 min, 7 days, and 30 days post-surgery. The percentage change of $\mathrm{rCBF}$ at each post-surgery time point was calculated by comparing the mean signal intensity to that of the baseline. Since isoflurane might have effects on $\operatorname{rCBF}[25,26]$, we have maintained isoflurane administration time and the concentration consistent for each animal to avoid potential confounding effects in each group. Additionally, body temperature was maintained at $36.5 \pm 0.5^{\circ} \mathrm{C}$ with a heating pad during surgery.

\section{Neurological behavioral function tests}

Neurologic function tests in mice were conducted in a blinded manner at 28-30 days after surgery, which includes the open field (OF) test to assess the locomotor activities and the Y-maze test to assess spatial working memory. In the Y maze test: each mouse was placed in a clear polycarbonate arena in the shape of a Y consisting of $3 \mathrm{arms}$ that were $33.65 \mathrm{~cm}$ long, $15 \mathrm{~cm}$ high, and $6 \mathrm{~cm}$ wide (Muromachi Kikai, Tokyo, Japan), and the mouse's behavior during free exploration of the $\mathrm{Y}$ maze was recorded for $8 \mathrm{~min}$ [27]. Behavioral tracking software (Noldus Ethovision XT) was used to analyze spontaneous alterations. The percentage of spontaneous alterations was calculated as the ratio of actual to possible alterations [defined as the frequency of spontaneous alteration behavior/(the total number of arm entries -2$) \times 100]$. In the OF test: each mouse was placed in the center of a square arena of open field apparatus $(40 \times 40 \times 40 \mathrm{~cm}$; Omnitech Electronics $)$ within environmental control chamber $(60 \times 64 \times 60 \mathrm{~cm}$; Omnitech Electronics). Total distance traveled (in $\mathrm{cm}$ ), vertical activity (rearing measured by counting the number of photobeam interruptions), and margin time (time spent in the periphery of the arena) were recorded using behavioral tracking software (Fusion, Omnitech Electronics). Data were collected for $60 \mathrm{~min}$.

\section{Magnetic resonance imaging DTI of ex vivo brain}

Post neurological behavioral tests, mice were anesthetized with $3 \%$ isoflurane in $70 \% \mathrm{~N}_{2} \mathrm{O}$ and $30 \% \mathrm{O}_{2}$, and transcardially perfused with $4 \%$ paraformaldehyde (PFA) and decapitated [28]. Brains were maintained within the skull to avoid anatomical deformation and fixed in $4 \%$ PFA overnight, then stored in PBS solution at $4{ }^{\circ} \mathrm{C}$. MRI was performed at $500 \mathrm{MHz}$ using a Bruker AV3HD 11.7 T/89 mm vertical bore small animal MRI scanner, equipped with a $20-\mathrm{mm}$ quadrature radiofrequency (RF) coil and Paravision 6.0.1 software (Bruker Biospin, Billerica, MA). Following positioning and pilot scans, T2weighted images (T2WI) were acquired using a Rapid Acquisition with Relaxation Enhancement (RARE) sequence, with the following parameters: Echo Time/Repetition Time $(\mathrm{TE} / \mathrm{TR})=20 / 4000 \mathrm{~ms}$, averages $=8,160 \times$ 160 matrix, 25 slices with a $0.5 \mathrm{~mm}$ slice thickness, a RARE factor $=4$, and a field of view (FOV) of $16 \times 16$ $\mathrm{mm}$. Hippocampal atrophy or signal abnormality (low or high signal intensity) were identified as injury on T2weighted images by one expert in small animal MRI imaging. A diffusion tensor imaging (DTI) data set covering the entire brain was collected using a multislice spin echo sequence with five reference and 30 non-collinear diffusion-weighted images with the following parameters: $\mathrm{TE} / \mathrm{TR}=22 / 2800 \mathrm{~ms}$, two averages, matrix size $=$ $160 \times 160$, field of view $=16 \times 16 \mathrm{~mm}, 25$ axial slices, slice thickness $=0.5 \mathrm{~mm}, b$ value $=3000 \mathrm{~s} / \mathrm{mm}^{2}$, and $\Delta /$ $\delta=11 / 5$ ms. MRI DTI data were analyzed with DSI Studio (http://dsistudio.labsolver.org/). In a blinded manner, regions of interest (ROIs) were drawn from the corpus callosum (CC), external capsule (EC), and hippocampus. Fractional anisotropy (FA), axonal diffusivity (AD), radial diffusivity (RD), and mean diffusivity (MD) values were determined for each ROI from identical consecutive sections of DTI images.

\section{Immunofluorescence staining image collection and IMARIS 3D morphological analysis of astrocytes}

Post-MRI PFA-fixed brains were equilibrated in $30 \%$ sucrose at $4{ }^{\circ} \mathrm{C}$ and coronal brain sections $(25 \mu \mathrm{m})$ were prepared using a cryostat (Leica SM2010R, Biosystems). After incubation with a blocking buffer for $1 \mathrm{~h}$ at room temperature, sections were incubated with primary antibodies against MBP (Rabbit, 1:800 dilution), NF-200 (Rabbit, 1:800 dilution), NHE1 (Rabbit; 1:100 dilution), 
GFAP (Mouse; 1:200 dilution), Iba1 (Goat; 1:600 dilution), NeuN (Mouse; 1:200 dilution), Phospho-p47phox (Rabbit; 1:100 dilution), or Lcn2 (Rat; 1:200 dilution) in blocking solution at $4{ }^{\circ} \mathrm{C}$ overnight. These brain sections were washed and incubated with secondary antibodies (1:200 dilution) for $1 \mathrm{~h}$ at room temperature. Subsequently, these sections were washed and incubated with DAPI (4,6-diamino-2-phenylindole, 1:1000 in 0.1M PBS) for $15 \mathrm{~min}$ at room temperature. Sections were then mounted on slides with a mounting medium. Fluorescence images of hippocampus overview were obtained under 10x objective by the Olympus IX83 inverted microscope (Olympus, Tokyo, Japan) and processed with Olympus cellSens Dimension software (version 2.3, Olympus). The fluorescence images from a $40 \times$ oilimmersion objective were captured using the Nikon A1R confocal microscope (Nikon, Tokyo, Japan) with NISElements AR software (version 4.51, Nikon). Images were obtained from identical slides positions using identical digital imaging acquisition parameters. Numbers of positively stained cells and intensity of immunoreactivity were quantified from the $40 \times$ oil-immersion objective images using the ImageJ software. Intensity of immunoreactivity was quantified by measuring the mean gray values and the results were expressed in arbitrary units.

For the 3D reconstruction and morphological quantitative analysis of reactive astrocytes, Bitplane Imaris software (Version 9.7.2, Bitplane, Zurich, Switzerland) was used. $Z$-stack images of GFAP $^{+}$astrocytes $(18-\mu \mathrm{m}$ depth, $1.69-\mu \mathrm{m}$ steps, $\times 40$ magnification) were taken using a Nikon A1R confocal microscope $(1024 \times 1024$ pixel, pixel size $0.16 \mu \mathrm{m}$ ). Raw images were converted using IMARIS converter (Version 9.7.2, Oxford Instruments). Images were subjected to surface and filament reconstruction based on GFAP immunostaining in three dimensions (3D). Surface reconstruction parameters were set to appropriately label all $\mathrm{GFAP}^{+}$astrocytes. The astrocyte processes and the voxels within one stack were rendered into 3D objects and the volume was analyzed. The cell body volume of the obtained objects was expressed as summated soma volume. The IMARIS Filament module was used to quantify morphological changes of astrocytic processes using the following endpoints: summarized process volume, mean diameter, and total terminal points of process. All images used for analysis were taken with the same confocal settings.

\section{Astrocyte isolation and RNA extraction for RNA sequencing}

In order to investigate the transcriptomic changes of astrocytes in response to BCAS and post-BCAS HOE642 treatment, sham, BCAS+Veh, and BCAS+HOE642 (i.p.) mice were harvested for RNA-seq (4 mice/group) and RT-qPCR (6 mice/group). At 30 days post-surgery, brains were removed and rapidly dissected in an ice-cold D-PBS solution. Single-cell suspensions were prepared from both hemispheres (without the cerebellum and brain stem) using the Adult Brain Dissociation Kit (Miltenyi Biotec, Germany), as described previously [29]. Hemispheres were separated in an enzyme mixture solution with gentle MACS Octo Dissociator at $37^{\circ} \mathrm{C}$ for 30 min. Digested tissues were filtered through a $70-\mu \mathrm{m}$ MACS Smart Strainer and followed by several steps of centrifugation to obtain a single-cell suspension. Astrocytes were further isolated by magnetic bead separation using anti-ACSA-2 microbead kit (Miltenyi Biotec, Germany). The RNA of $\mathrm{ACSA}^{+}$astrocytes was extracted using the RNeasy Micro kit (Qiagen, 74004) following the manufacturer's protocol. The resulting RNA was eluted with RNase-free water and stored at $-80{ }^{\circ} \mathrm{C}$. Samples were sequenced on an Illumina NovaSeq 6000 (PE150) using Illumina TruSeq stranded mRNA kit for library preparation. Total RNA ( $300 \mathrm{ng}$ ) was used as input for library preparation.

\section{Bioinformatic data analysis}

RNA-Seq data were analyzed following the instruction of CLC genomics Workbench 21 (CLC bio, Aarhus, Denmark) [29]. Briefly, quality control was conducted for the paired-end reads in FASTQ format, before mapping against the mouse reference genome GRCm38 (mm10) using default parameters of the "RNA-Seq Analysis" tool. Gene and transcript annotations were completed with Ensembl (release V103). Differentially expressed genes (DEGs) were identified between sham, $\mathrm{BCAS}+\mathrm{Veh}$, and BCAS+HOE (i.p.) groups using the "Differential Expressions for RNA seq" tool. Genes with a $p$ value $\leq 0.05$ and fold change (FC) $\geq 1.5$ or $\leq-1.5$ were identified as differentially expressed genes. QIAG EN's Ingenuity Pathway Analysis (IPA', QIAGEN Redwood City, www.qiagen.com/ingenuity) was used to identify statistically enriched biological pathways associated with the differentially expressed genes. Statistical significance was calculated using the right-tailed Fisher's exact probability tests; biological pathways showing $p$ value $<0.05$ were considered statistically significant. The activity status of pathways was determined by calculating the activity $Z$-score, a statistical measure of how closely the gene expression pattern present in the query dataset compares to the expected pattern based on the literature findings [30]. A positive score indicates an overall increase in the pathway activity, whereas a negative value indicates an overall decrease in activity. The IPA Comparison Analysis tool was used to compare pathway enrichment analysis results generated from the multiple datasets used in our study. A $p$ value $<0.05$ and a $Z$ score $\geq 2$ were set as the thresholds for statistical significance. Gene ontology (GO) analyses were conducted for 
biological processes, using the Database for Annotation Visualization and Integrated Discovery (DAVID; https:// david.ncifcrf.gov/) [31], with $p$ value $<0.05$ and a gene count $\geq 2$ as the thresholds to indicate a statistically significant difference.

\section{RT-qPCR analysis}

RNA was extracted from MACS-isolated astrocytes using the RNeasy Micro kit (Qiagen, 74004) following the manufacturer's instructions. RNA was quantified by measuring absorbance with spectrophotometer ND-1000 (NanoDrop). Reverse transcription was performed using the iScript Reverse Transcription Supermix (Bio-Rad) according to the manufacturer's protocol. All RNA isolated from cell pellets was converted into cDNA. Quantitative RT-PCR was performed using iTaq Universal SYBR Green Supermix (Bio-Rad) on a CFX 96 Touch Real-Time PCR Detection System. All relative gene expression analyses were performed using the $2^{-\Delta \Delta \mathrm{Ct}}$ method with duplicate reactions for each evaluated gene. Following primer sequences were used: Hprt (housekeeping gene), forward: GCC TAA GAT GAG CGC AAG TTG, reverse: TAC TAG GCA GAT GGC CAC AGG; Ptgs2, forward: TGA GCA ACT ATT CCA AAC CAGC, reverse: GCA CGT AGT CTT CGA TCA CTA TC; Nos3, forward: TCA GCC ATC ACA GTG TTC CC, reverse: ATA GCC CGC ATA GCG TAT CAG; Lnc2, forward: TGG CCC TGA GTG TCA TGTG, reverse: CTC TTG TAG CTC ATA GAT GGT GC; $M m p 9$ forward: GGA CCC GAA GCG GAC ATT G, reverse: CGT CGT CGA AAT GGG CAT CT. The data were normalized to Hprt as a reference gene.

\section{Statistical analysis}

Mice were coded with randomized numbers and outcome assessments were performed by investigators who were blinded to the treatment conditions. A total of 69 mice were used and all data were included except two outlier samples excluded in RNA-seq bioinformatics analysis after inspection of a PCA bi-plot and data. Normality was assessed by the Shapiro-Wilk test. Data were presented as mean and standard deviation (SD) if data were normally distributed, or reported as median and quartiles if data were not normally distributed. Statistical significance was determined by Student's $t$-test or oneway analysis of variance (ANOVA) followed by Bonferroni post hoc test. The repeated measured values within groups were analyzed by repeated measure ANOVA followed by Bonferroni's post hoc test. The GraphPad Prism software was used for statistical analyses (GraphPad Software, Inc., CA, USA). The Pearson correlation analysis and ANOVA analysis followed by the LSD post hoc test for DTI metrics were performed using SPSS 24
(SPSS Inc., Chicago, Ill., USA). A $p$ value $<0.05$ was considered statistically significant.

\section{Results \\ Effects of NHE1 blockade on BCAS-induced changes in $\mathrm{rCBF}$ and cognitive function impairment}

C57BL/6J mice were subjected to sham or BCAS surgery, or with subsequent treatment regimens including BCAS+Veh (i.p.), BCAS+HOE642 (i.p.), or BCAS+ HOE642 (pump) (Fig. 1a). PeriCam laser speckle contrast analysis shows that sham mice displayed no significant changes in $\mathrm{rCBF}$ from prior to surgery through 30 days post-surgery $(p>0.05$, Fig. $1 \mathrm{~b}, \mathrm{c})$. In contrast, the $\mathrm{BCAS}+\mathrm{Veh}$ mice and BCAS+HOE (i.p.) mice displayed $\sim 45 \% \mathrm{rCBF}$ reduction at the onset of BCAS, which gradually recovered to $\sim 75 \%$ of baseline by 30 days post-surgery ( $p<0.05$, Fig. 1b, c). However, the BCAS+ $\mathrm{HOE}$ (pump) mice showed a trend of less $\mathrm{rCBF}$ reduction at onset, 7 days, or 30 days after BCAS surgery, compared to the BCAS+HOE (i.p.) group, but did not reach statistical significance $(p>0.05$, Fig. $1 b, c)$. No differences in $\mathrm{rCBF}$ were detected between the BCAS+Veh mice and BCAS+HOE (i.p.) mice $(p>0.05$, Fig. 1b, c).

We then evaluated neurological function changes in these mice with $\mathrm{Y}$ maze and OF tests. In the $\mathrm{Y}$ maze test, no differences in total entry counts were detected among all four testing groups, indicating similar locomotor activity in these mice ( $p>0.05$, Fig. 1d). However, a significantly lower alternation rate, indicative of spatial working memory deficit [32], was observed in the BCAS+Veh group, compared with the sham group $(p<$ 0.05 , Fig. 1d). Interestingly, the BCAS+HOE (i.p.) group displayed similar spontaneous alternation rates as the sham group, showing no spatial working memory deficit. The BCAS+HOE (pump) mice performed significantly better than the BCAS+Veh group but worse than the BCAS+HOE (i.p.) group $(p<0.05$, Fig. 1d). In the OF test, no significant differences in distance traveled or margin time (data not shown) were detected among the four testing groups (Fig. 1e). However, the BCAS+Veh group exhibited a trend of higher vertical activities, compared with the sham group, but did not reach statistical significance $(p>0.05)$. The BCAS+HOE (pump) mice showed occasional lower locomotor activity, e.g., less traveled distance than sham mice at the initial $5 \mathrm{~min}$ and less vertical activity at $30 \mathrm{~min}$ than the BCAS+Veh mice ( $p<0.05$, Fig. 1e). Taken together, these data demonstrated that BCAS-induced cerebral hypoperfusion in mice impaired their spatial working memory. Pharmacological blockade of NHE1 protein with NHE1 inhibitor HOE642 (daily i.p. injection or continuous mini pump delivery) effectively prevented or attenuated BCASinduced cognitive function impairment. 


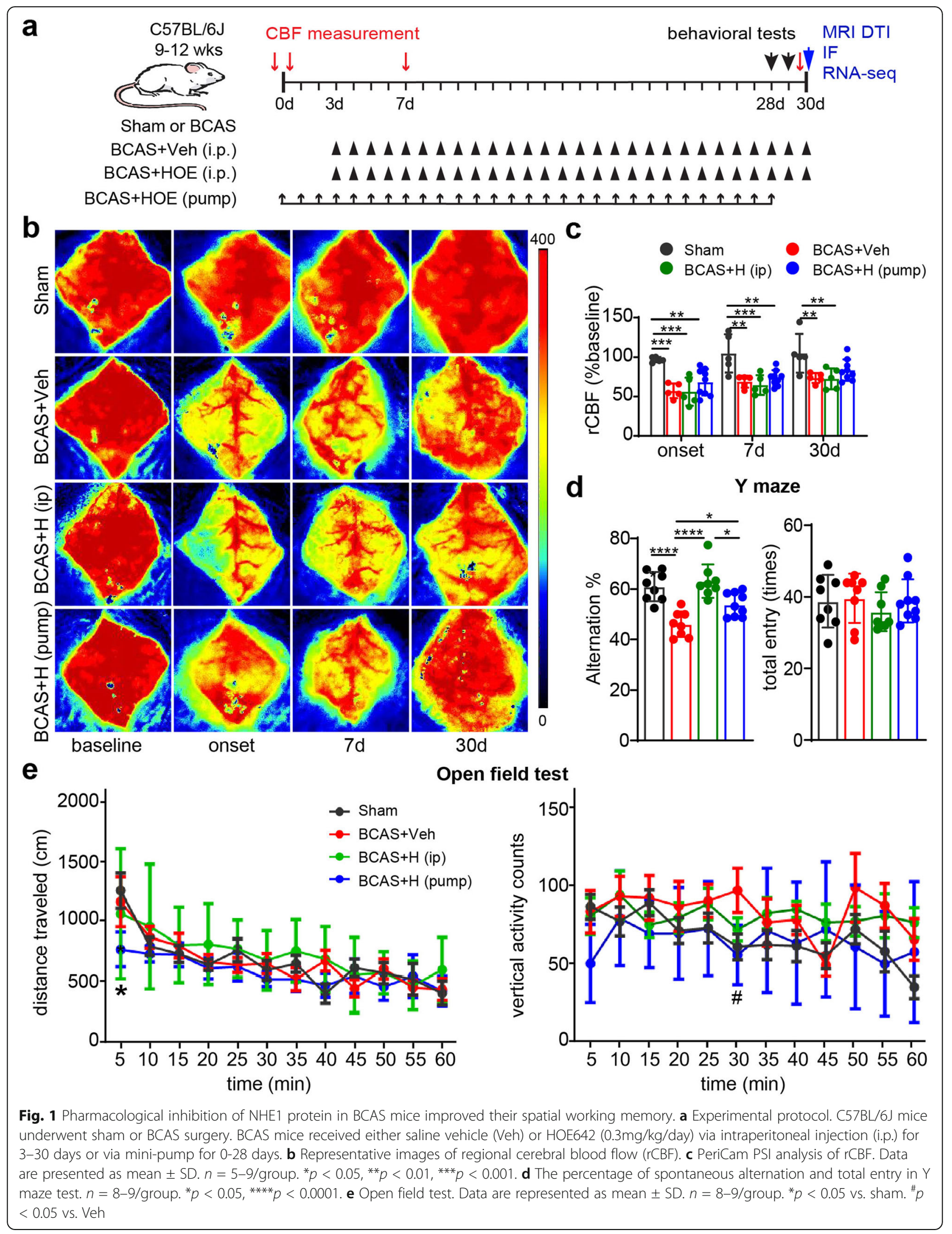


Pharmacological inhibition of NHE1 protein attenuated BCAS-induced white matter tract damage

To understand the mechanisms underlying neurological function protection in the HOE642-treated mice, ex vivo MRI studies were performed on brains from these four groups of mice (sham, BCAS+Veh, and BCAS+HOE mice (i.p., or pump). Representative directionally encoded color (DEC) maps, FA maps, and T2-weighted images showed no obvious white matter lesions in the four groups (supplemental Fig S1a). However, DTI metrics measurement and analysis of white matter tracts (Fig. 2a), such as corpus callosum (CC) and external capsule (EC), revealed an average of $15 \%$ reduction in the mean FA values in CC ( $p<0.05$, Fig. $2 b)$ and $11.2 \%$ reduction in $\mathrm{EC}(p<0.05$, Fig. $2 \mathrm{~b})$ in the BCAS+Veh brains, compared to sham brains. In contrast, the BCAS+HOE-treated brains (i.p. or pump) showed significantly higher FA values in $C C$ and $E C$ than the BCAS+Veh brains $(p<0.05$, Fig. $2 b)$. These non-biased DTI findings indicate that pharmacological blockade of NHE1 protein preserved white matter integrity, which may contribute to improved neurological function outcomes. This speculation is supported by immunofluorescence staining of these brains for assessment of demyelination, shown by loss of myelin basic protein (MBP) and axonal neurofilament (NF-200) expressions. Due to a lack of optimal anti-MBP and NF200 antibodies for double labeling, the images in Fig. 2c were obtained from anti-MBP or anti-NF200 staining with DAPI respectively. Compared to sham or naïve brains (supplemental Fig. S2), reduction of MBP (40-48\%) and NF expression (44-47\%) was detected in CC and EC of the BCAS+Veh brains $(p<0.05$, Fig. $2 \mathrm{c}, \mathrm{d})$ or the BCAS mice (supplemental Fig. S2). In contrast, the BCAS+ HOE-treated mice (i.p. or pump) showed a trend of higher MBP or NF-200 expression in CC and EC than the BCAS+Veh mice $(p>0.05$, Fig. $2 c, d)$. The FA values were highly correlated with NF expression in CC and EC (with the Pearson coefficient $r=0.66-0.80, p<0.05$, Table S1). Taken together, these results demonstrate that inhibition of NHE1 activity with HOE642 ameliorated BCAS-induced demyelination and axonal damage in white matter tracts.

\section{Pharmacological inhibition of NHE1 protein reduced BCAS-induced astrogliosis in white matter tissues}

Astrogliosis is a characteristic pathology in VCID and BCAS-induced white matter lesions [11, 12, 14]. We analyzed changes in $\mathrm{GFAP}^{+}$astrocytes in the CC and EC white matter tracts from post-MRI ex vivo brains. Low numbers of $\mathrm{GFAP}^{+}$cell counts were detected in sham brains (Fig. 3a-d), with basal levels of GFAP and NHE1 protein expression (arrow) in homeostatic astrocytes, which displayed small soma and fine processes (arrow,
Fig. 3a, c). In contrast, the BCAS+Veh brains exhibited significantly higher GFAP intensity and increased $\mathrm{GFAP}^{+}$cell counts in the CC and EC $(p<0.05$, Fig. 3ad). The IMARIS analysis of 3D structural changes of reactive astrocytes showed that compared to sham brains, $\mathrm{GFAP}^{+}$astrocytes in the BCAS+Veh brains displayed significantly larger soma volume and process diameter in $\mathrm{CC}$, along with more process terminal points, indicating hypertrophy of reactive astrocytes (arrows, $p<0.05$, Fig. $4 \mathrm{a}, \mathrm{b})$. However, the 3D structures of reactive astrocytes in the BCAS+HOE brains exhibited astrocytic morphology similar to sham brains, with smaller soma volume and process diameter or less process terminal points. Similar patterns of morphologic changes of astrocytes in EC among these three groups were also detected (data not shown). In addition, we also detected an increased number of $\mathrm{Iba}^{+}$microglia, some of which showed amoeboid morphology in the CC and EC of the BCAS+ Veh brains (supplemental Fig. S3a, b), and colocalization with NHE1 protein (arrow, supplemental Fig. S3a). Collectively, these data indicate that blocking NHE1 protein with HOE642 reduces both astrogliosis and microglial activation in white matter, which likely prevented BCAS-induced demyelination.

\section{Effects of NHE1 blockade on the BCAS-induced microstructural changes of hippocampus}

As the $Y$ maze spontaneous alternation rate indicates for spatial working memory, which requires hippocampal integrity [33], we examined whether HOE642 treatment affected BCAS-induced damage in the hippocampus. No significant $\mathrm{NeuN}^{+}$neuron loss (supplemental Fig S4) or changes in DTI parameters in the sensorimotor cortices were detected among the four experimental groups (data not shown). Ex-vivo T2-weighted MRI showed slight injury to the hippocampus of the BCAS+Veh and BCAS+ HOE (pump) mice (double arrow, Fig. 5a). In contrast, DTI metrics measurement revealed that the whole hippocampus (from bregma - 0.94 to $-4.04 \mathrm{~mm}$ in both hemispheres) of the BCAS+Veh mice exhibited significantly lower FA values but higher RD values, and a trend of higher MD values, compared to sham brains $(p<$ 0.05 , Fig. 5b, c). The reduced FA along with increased MD and RD have been broadly reported to be associated with demyelination [34]. In further analysis of different hippocampal regions, the BCAS+Veh brains displayed higher $\mathrm{MD}$ and $\mathrm{RD}$ values in the stratum lacunosum moleculare (slm) and molecular layer $(\mathrm{ml})$ of the dentate gyrus $(p<0.05$, Fig. $5 \mathrm{~b}-\mathrm{d})$. Interestingly, the BCAS+ HOE (pump) mice exhibited values of DTI metrics (either in the whole hippocampus or its strata) that are comparable with sham mice ( $p>0.05$, Fig. $5 b-d)$. But, the BCAS+HOE (i.p.) group showed similar DTI metrics (FA, RD and MD values) with the BCAS+Veh mice. In 


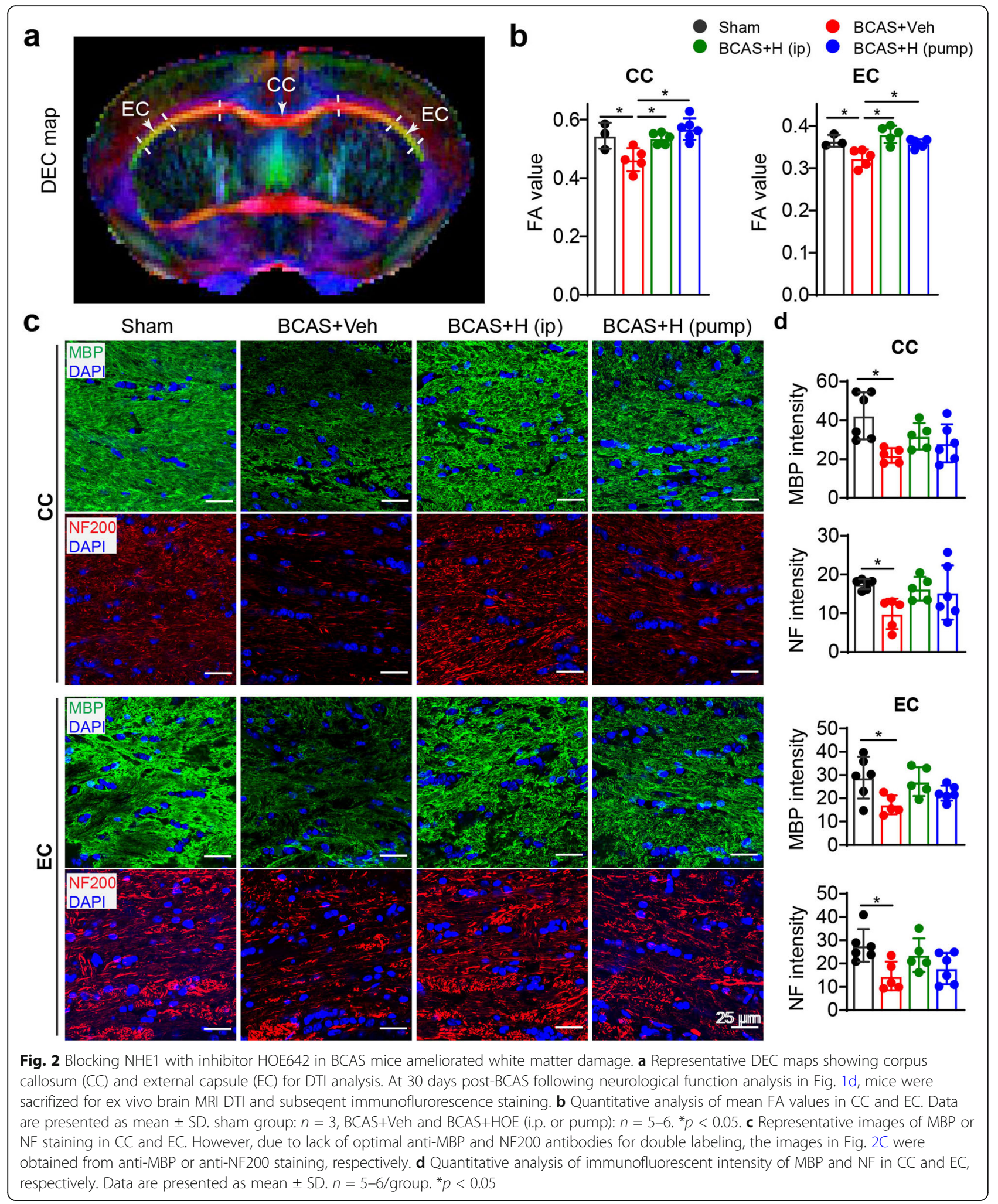

assessing hippocampal gliosis in these brains with immunostaining of $\mathrm{GFAP}^{+}$reactive astrocytes, we detected substantial reactive astrogliosis in the hippocampus of the BCAS+Veh brains (arrow, Fig. 6a), with a significant increase in GFAP intensity in the stratum radiatum (sr) of the hippocampus and an increase in $\mathrm{GFAP}^{+}$astrocytes, albeit insignificant, in polymorphic layer (pl) of the dentate gyrus (arrow, Fig. 6b, c). In contrast, the 


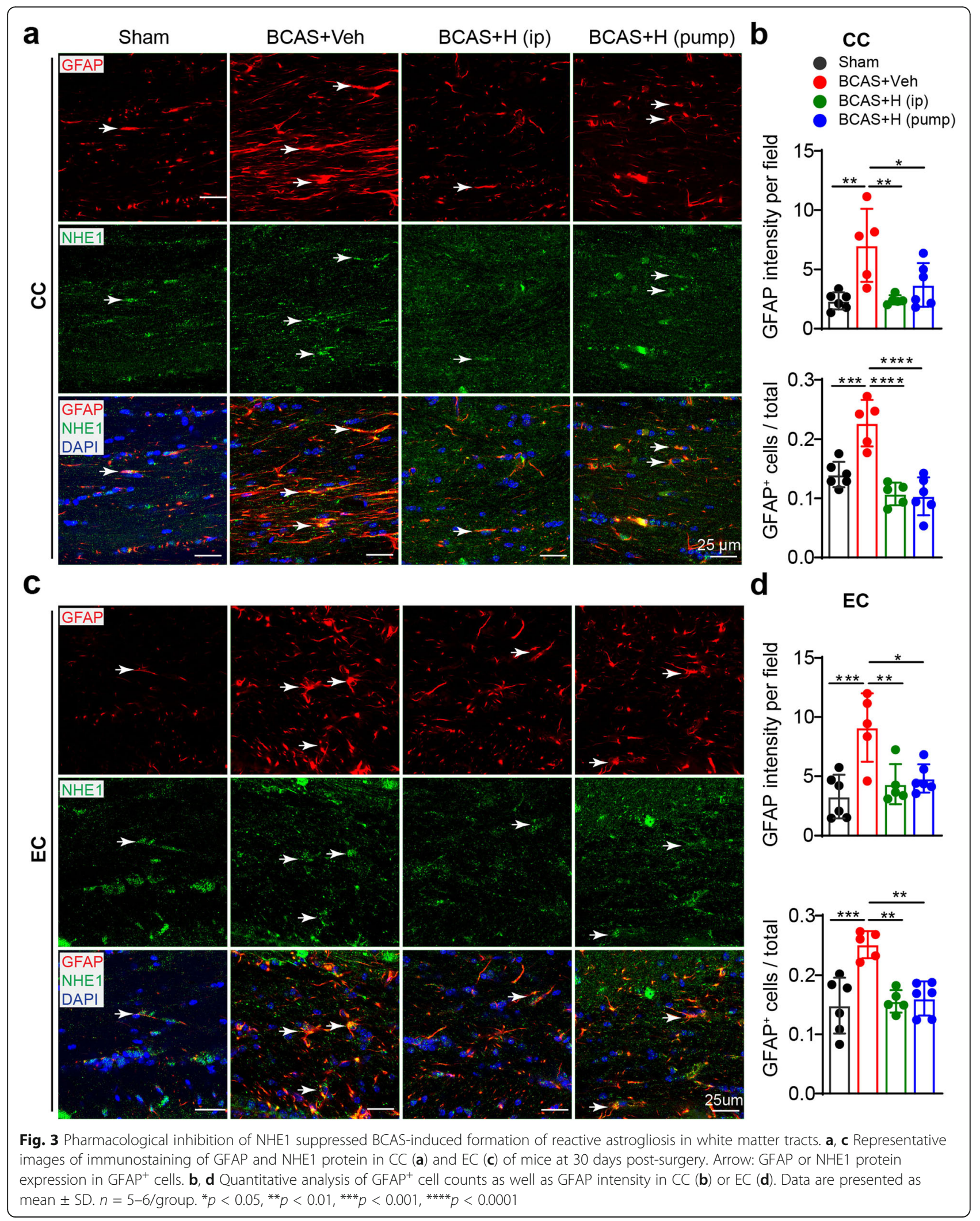




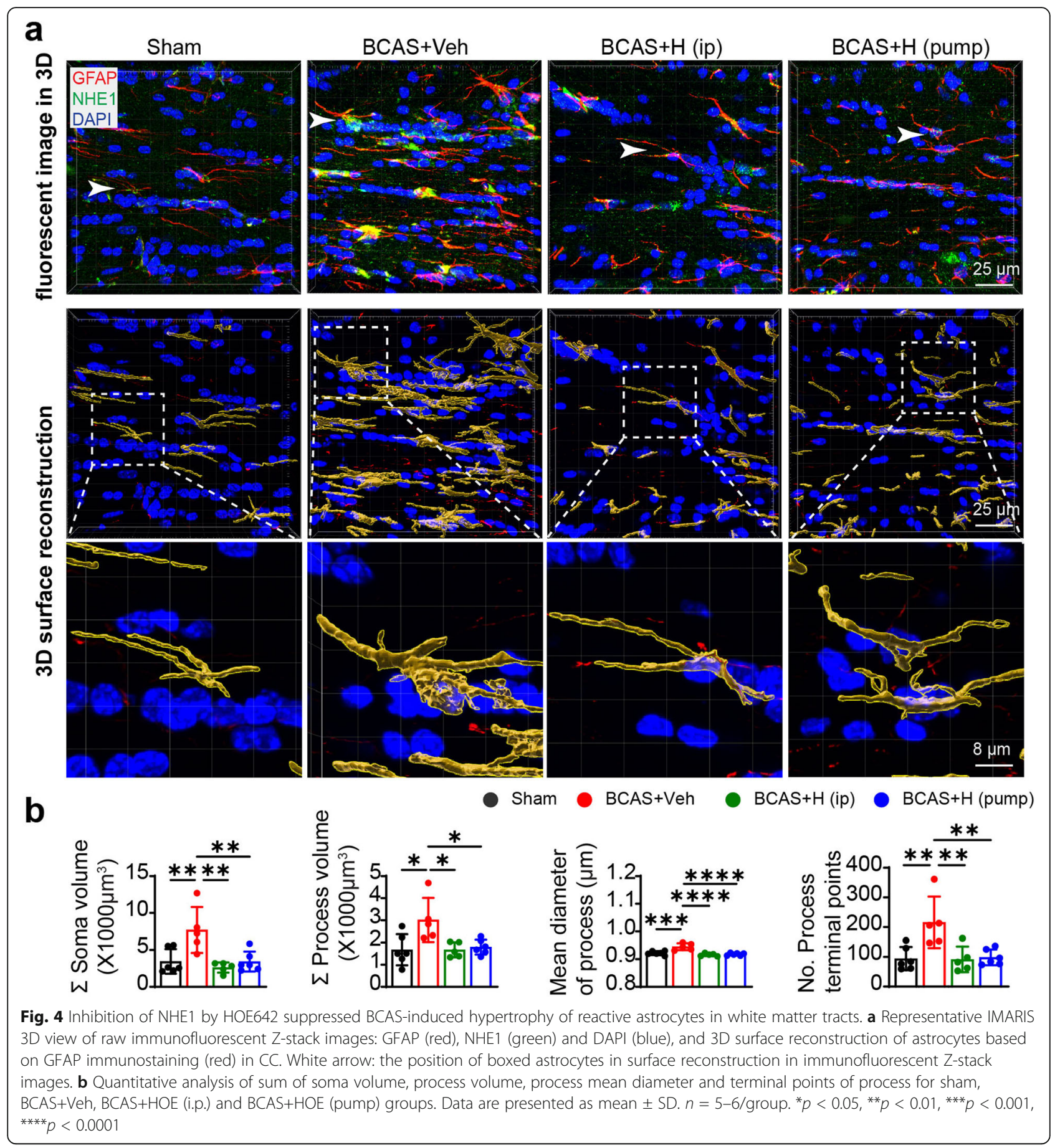

BCAS $+\mathrm{HOE}$ (i.p.) group exhibited attenuated reactive $\mathrm{GFAP}^{+}$astrocytes in sr compared with the BCAS+ Veh mice $(p<0.05$, Fig. $6 \mathrm{~b}, \mathrm{c})$, but this change was not detected in the sr of the hippocampus in the BCAS+HOE (pump) mice (Fig. 6b, c). Additionally, the BCAS+HOE-treated brains (i.p. or pump) also exhibited the absence of activation of microglia in the sr of the hippocampus (Supplemental Fig. S5a-c). Taken together, inhibition of NHE1 protein with
HOE642 attenuated BCAS-induced gliosis in the hippocampus, which likely contributes to preserving hippocampal integrity and cognitive function. Considering the contributions of astrogliosis to increasing FA and decreasing RD values in DTI [35], the discrepancy in hippocampal astrogliosis and changes of DTI metrics in the BCAS+HOE-treated brains (i.p. or pump) is addressed in the "Discussion" section. 


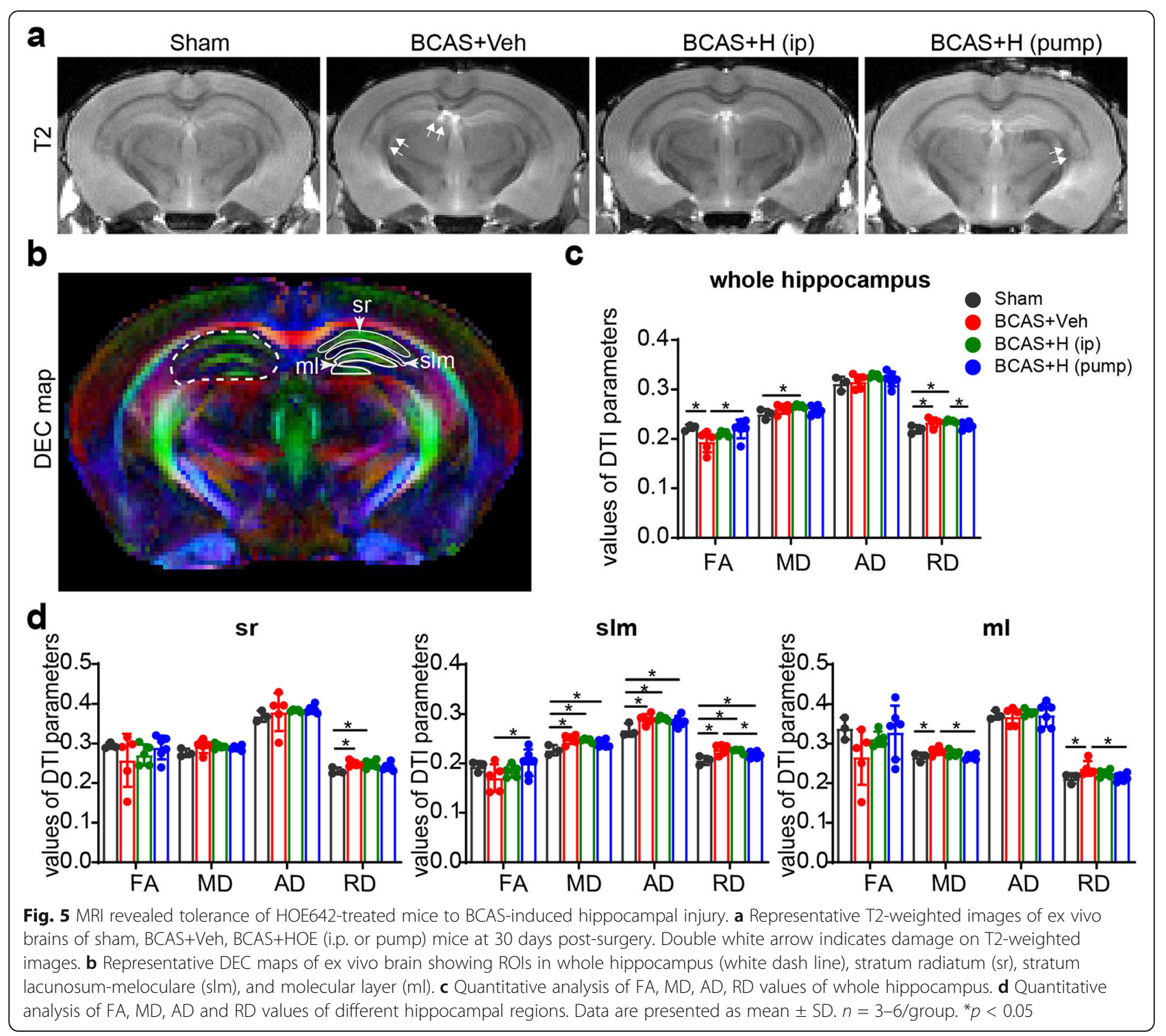

Correlations of cognition function and structural integrity of white matter and hippocampus

We conducted Pearson's correlation analysis to explore the relationships between BCAS-induced gliosis, white matter or hippocampal structural damage, and mouse cognitive function deficits. We found that the alternation rate of mice in $\mathrm{Y}$ maze test was correlated with FA value change in $\mathrm{CC}$ $(r=0.473, p<0.05)$ and EC $(r=0.64, p<0.05)$ (Fig. 7a, b and supplemental Table S1). At the cellular level, elevation of reactive $\mathrm{GFAP}^{+}$astrocytes in $\mathrm{CC}(r=-0.733)$ or EC ( $r=$ - 0.609) and activated Iba1 ${ }^{+}$microglia in CC $(r=-0.622)$ or EC $(r=-0.653)$ were negatively correlated with spatial working memory of mice in Y maze test (Fig. 7c, d and supplemental Table S1). In the hippocampus, GFAP ${ }^{+}$astrocyte counts and $\mathrm{Iba}^{+}$counts in the sr or pl of the hippocampus significantly correlated with the alternation rate in $\mathrm{Y}$ maze test (Fig. 7e, f and supplemental Table S1). The alternation rate in $\mathrm{Y}$ maze task was also significantly correlated with $\mathrm{RD}$ in slm and $\mathrm{ml}$ of hippocampus ( $r=0.55-0.6$, supplemental Table S1), although we did not detect a correlation between cognitive function and DTI metrics of the whole hippocampus. Taken together, these findings suggest that glial activation in white matter tracts and hippocampus is closely associated with spatial working memory impairment induced by BCAS. Pharmacological inhibition of NHE1 protein attenuates glial activation, which led to the preservation of white matter integrity and hippocampal integrity and improvement of spatial working memory.

Pharmacological inhibition of NHE1 protein revealed reduced transcriptome profiles of ROS production and inflammatory responses in astrocytes

To understand the underlying mechanisms of NHE1 protein in astrogliosis in BCAS brains, we conducted an 


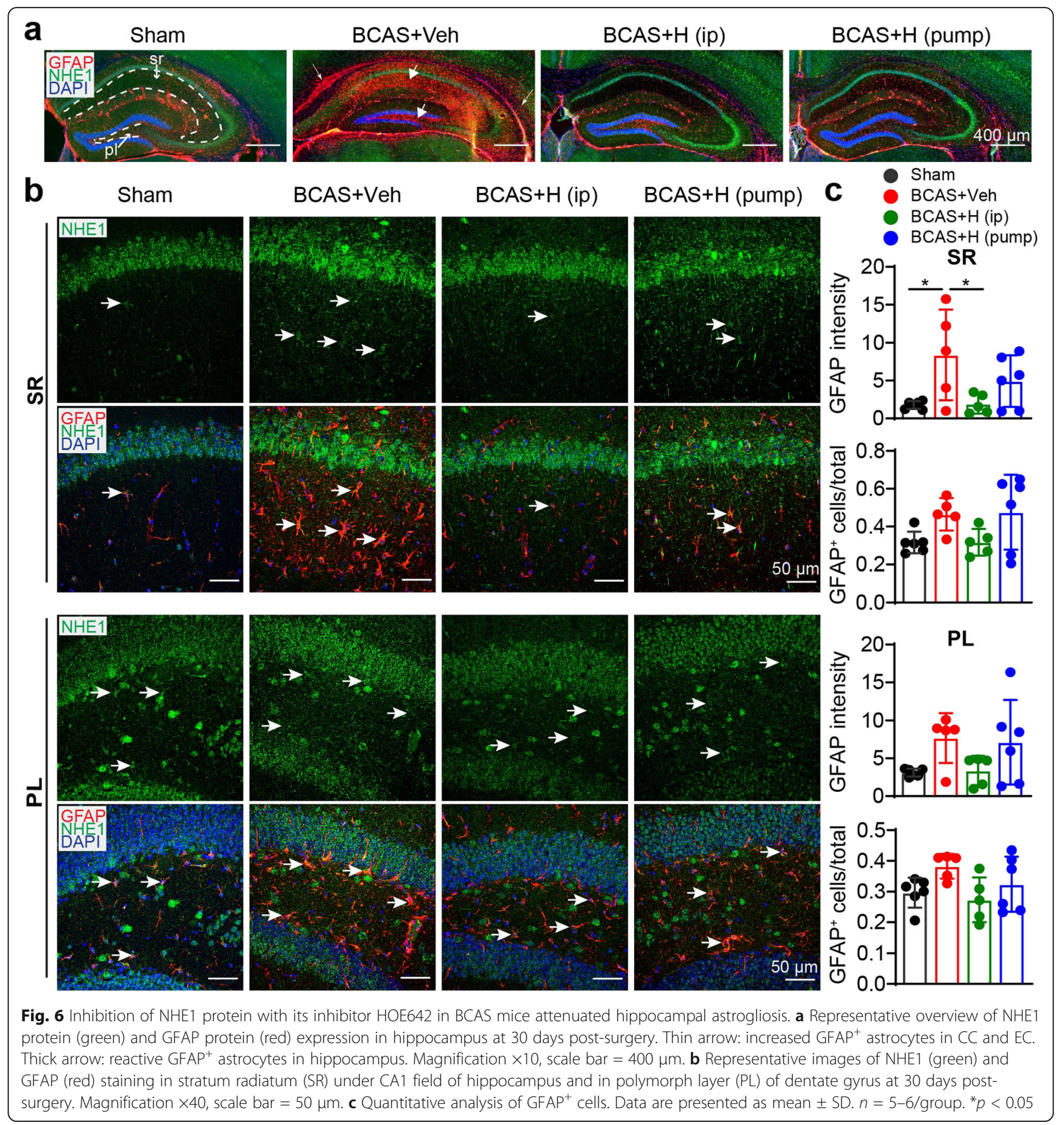

RNA-seq of MACS-isolated brain astrocytes from sham, BCAS+Veh (i.p.), and BCAS+HOE (i.p.) mice (Fig. 8a). The purity of the isolated $\mathrm{ASCA}^{+}$astrocytes was validated by their highly expressed gene markers Atp $1 b 2$, Slc2a1, Aqp4, Gfap, Aldh1l1 that are specifically enriched in astrocytes (Supplemental Fig. S6). Specifically, no marker genes for neurons and only trace levels of specific marker genes for microglia, oligodendrocytes, or endothelial cells were detected. The bulk RNA-seq analysis identified 1130 differentially expressed genes in the $\mathrm{BCAS}+\mathrm{HOE}$-treated brain astrocytes, compared with the BCAS+Veh-treated brain astrocytes (Fig. 8b), including 340 upregulated genes and 790 downregulated genes (Fig. 8c). GO analysis using DAVID software showed that the upregulated genes were involved in neuroprotective biological processes, such as "signal transduction," "cell adhesion," "multicellular organism development," "nervous system development," "myelination," and "ion transport" $(p<0.05)$ (Supplemental Fig. $\mathrm{S7}$ ), whereas the downregulated genes in the BCAS+ 


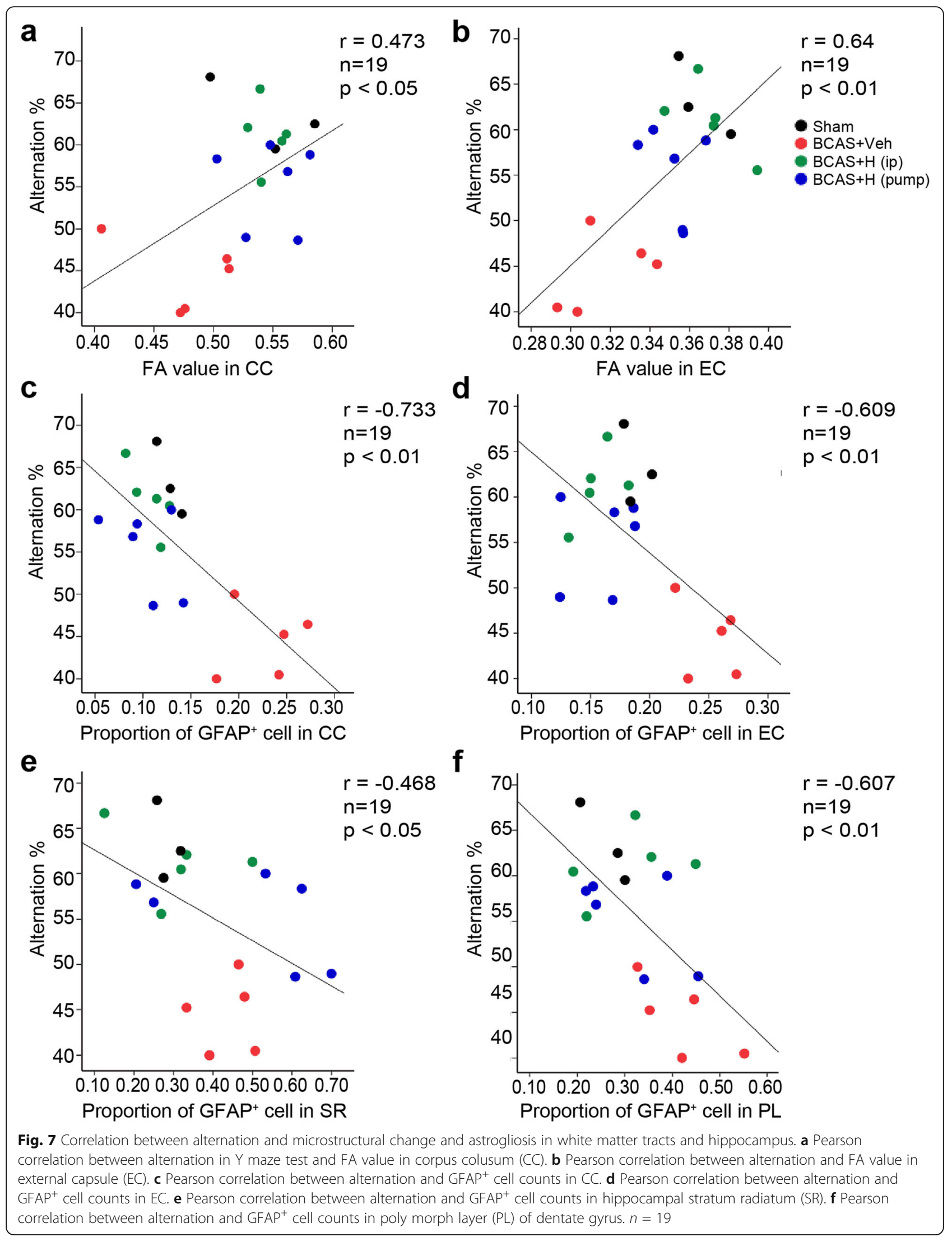




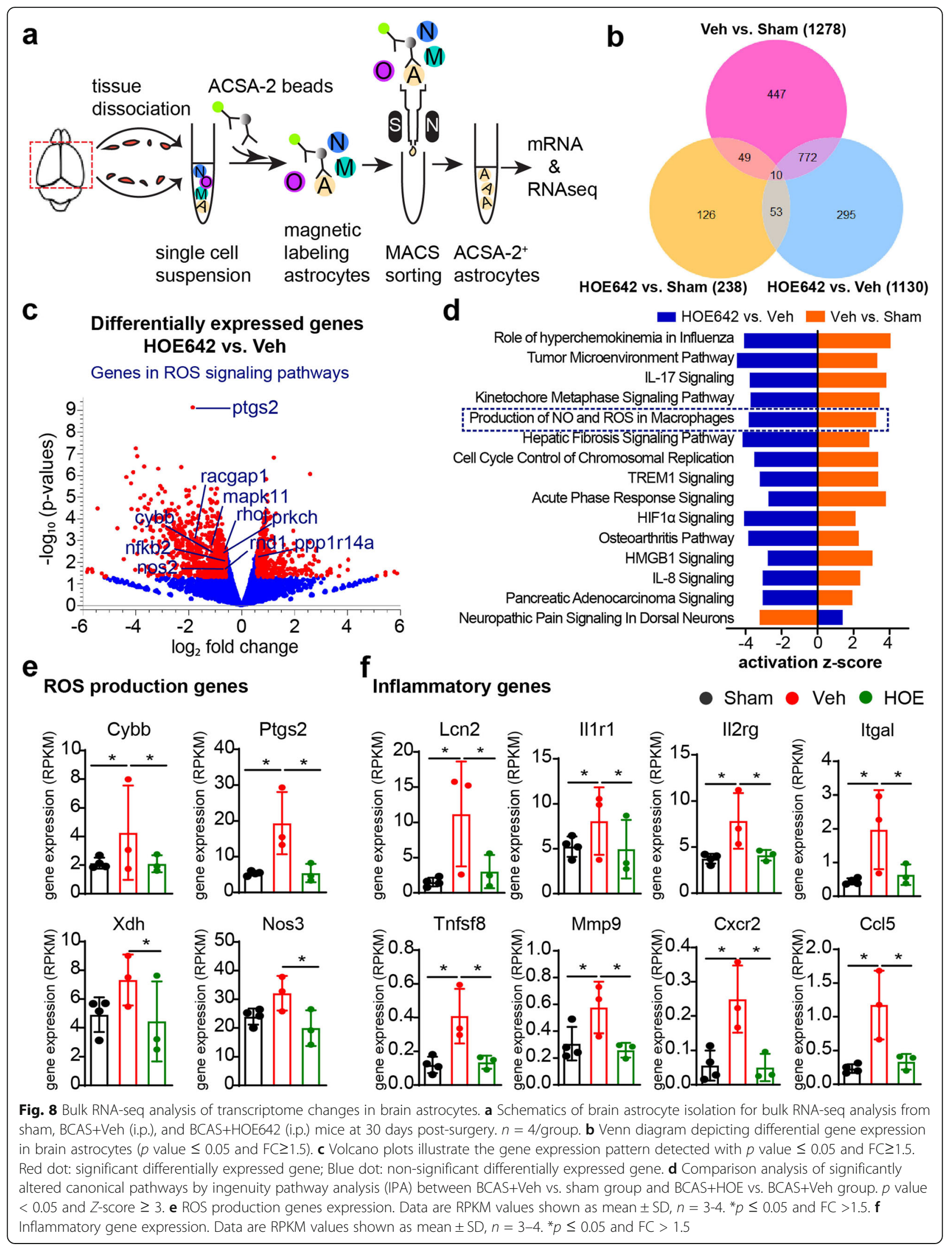


HOE-treated brain astrocytes were mainly involved in biological processes of "cell cycle" and "inflammatory responses" (Supplemental Fig. S8).

In light of diverse transcriptomic profiling of reactive astrocytes in different disease models [36, 37], we further explored the changes of reactive astrocyte transcriptomes in our study. In comparison to the sham controls, the BCAS+Veh astrocytes showed significant upregulation of classic transcriptomic signatures of reactive astrocytes, including pan-reactive signatures (Vim, Lcn2, Cd44, Steap4, Cxcl10, Gfap, Serpina3n, Osmr, Timp1), the reported clustered A1 phenotype genes ( $F k b p 5$, Gbp2, H2-D1, H2-T23, Iigp1, C3, C4b, Psmb8, Serping1, Srgn) or A2 phenotype genes (Cd14, Emp1, Ptgs2, Tgm1) ( $p \leq 0.05$ and $\mathrm{FC} \geq 1.5$, Supplemental Fig. S9). In contrast, the astrocytes isolated from the BCAS+HOEtreated mice presented transcriptome profiles that resembled the sham control astrocyte profile, e.g., with downregulated pan-reactive signatures (Vim, Lcn2, Cd44, Cxcl10, Hspb1, Gfap, Serpina3n, Osmr, Timp1), and lower expression level of the clustered genes (Gbp2, H2-D1, C3, C4b, Psmb8, Serping1, Srgn) and (Cd14, Emp1, Ptgs2, Tgm1) (supplemental Fig. S9). Collectively, these results demonstrated that BCAS triggered proinflammatory transcriptome changes of reactive astrocytes, and pharmacological inhibition of NHE1 prevented such changes in astrocytes.

In addition, the pathway enrichment analysis using IPA for DEGs reveals that BCAS induced activation of the inflammation and cell cycle-related pathways, compared with sham groups, and that HOE642 treatment inhibited these changes $(p<0.05$ and $Z$-score $\geq 3$, Fig. $8 \mathrm{~d})$. Of note, the "production of NO and ROS in macrophages" pathway was significantly elevated by BCAS while suppressed by HOE treatment (Fig. 8d). The gene $C y b b$ encoding NOX2 subunit gp $91^{\text {phox }}$ was upregulated in astrocytes of the BCAS+Veh mice but was significantly downregulated in the $\mathrm{BCAS}+\mathrm{HOE}$ mice $(p<0.05$ and $\mathrm{FC}>1.5$, Fig. $8 \mathrm{c}, \mathrm{e})$. In addition, BCAS triggered significant elevation of the expression of other reactive oxygen species (ROS) signaling related genes, including NOX2 subunit rac-related genes (Racgap1, Rhoj, Rnd1), Pro-inflammatory and ROS production enzyme cyclooxygenase-2 (COX2) gene (Ptgs2), ROS signaling downstream genes (Mapk11), and inflammatory genes (Lcn2, Il1r1, Il2rg, Tnfsf8, Mmp9, Cxcr2, Ccl5, Itgal, etc.). In contrast, HOE642 treatment prevented upregulation of these signature genes ( $p \leq 0.05$ and FC $>1.5$, Fig. $8 \mathrm{c}$, $\mathrm{e}$, and $\mathrm{f}$ and supplemental Fig. S10). In addition, HOE642 treatment significantly downregulated the NOX2 activator protein kinase $\mathrm{C}$ gene (Prkch), other ROS production genes (Nos2, Nos3, Xdh), and ROS signaling downstream transcription factor gene (Nfkb2) ( $p$ $\leq 0.05$ and $\mathrm{FC}>1.5$, Fig. 8c, e, and $\mathrm{f}$ and supplemental
Fig. S10). Taken together, the bioinformatic analysis of astrocytes revealed that inhibition of NHE1 protein with HOE642 prevented BCAS-induced astrocytic transcriptome changes for ROS and inflammatory cytokines release, which collectively drive attenuation of gliosis, demyelination, and cognitive dysfunction.

\section{NHE-1 blockade attenuated astrocytic NOX2 activation in white matter}

NHE1-mediated $\mathrm{H}^{+}$extrusion promotes NOX2 activation in microglia and neurons $[21,38]$. To further investigate that blockade of NHE1 protein reduced the ROS production and pro-inflammation resulting from attenuating NOX2 activity in white matter reactive astrocytes, we examined changes of expression of phosphorylated cytosolic subunit $\mathrm{p} 47$ ( $p$-p47 phox) of NOX2 in astrocytes in CC and EC white matter tracts, since phosphorylation of cytosolic subunits $\mathrm{p} 40, \mathrm{p} 47$, and $\mathrm{p} 67$ is required for NOX2 activation [39]. We also probed changes of LCN2 (Lipocalin 2) protein expression since it stimulates the classical proinflammatory reaction in astrocytes [40]. As shown in Fig. 9, compared with the sham group, the BCAS+Veh mice showed abundant expression of phosphorylated $\mathrm{p} 47$ phox ( $p$-p47) and LCN2 in astrocytes in $\mathrm{CC}$ and $\mathrm{EC}$ white matter tracts. The BCAS+HOE642 (i.p.) treatment suppressed phosphorylation of $\mathrm{p} 47$ phox and LCN2 protein upregulation in astrocytes $(p<0.05$, Fig. 9). Collectively, these results suggested the blockade of NHE1 protein inhibited ROS production and pro-inflammatory activation in astrocytes, which contributed to the attenuation of gliosis, demyelination, and cognitive dysfunction.

\section{Discussion}

BCAS-induced astrogliosis in the white matter and hippocampus and cognitive function deficits

$\mathrm{CCH}$ causes white matter lesions and hippocampal atrophy and is one of the major contributions to VCID [41, 42]. Among various VCID models, the BCAS model induces mild chronic hypoperfusion and simulates key characteristic pathogenesis of VCID, including white matter lesions, gliosis, inflammation [3]. BCAS triggers reactive astrogliosis in the white matter [32, 43], cortex, striatum, and hippocampus [44]. In our study, we detected substantial activation of glial cells $\left(\mathrm{GFAP}^{+}\right.$reactive astrocytes and $\mathrm{Iba1}^{+}$microglia) in the $\mathrm{CC}, \mathrm{EC}$, and hippocampus at 30 days post-BCAS, which were associated with white matter and hippocampal demyelination and microstructural damage. Importantly, chronic administration of NHE1 inhibitor HOE642 in BCAS mice significantly reduced cell counts of $\mathrm{GFAP}^{+}$reactive astrocytes and $\mathrm{Iba}^{+}$microglia in white matter as well as in the hippocampus. This reveals the therapeutic 


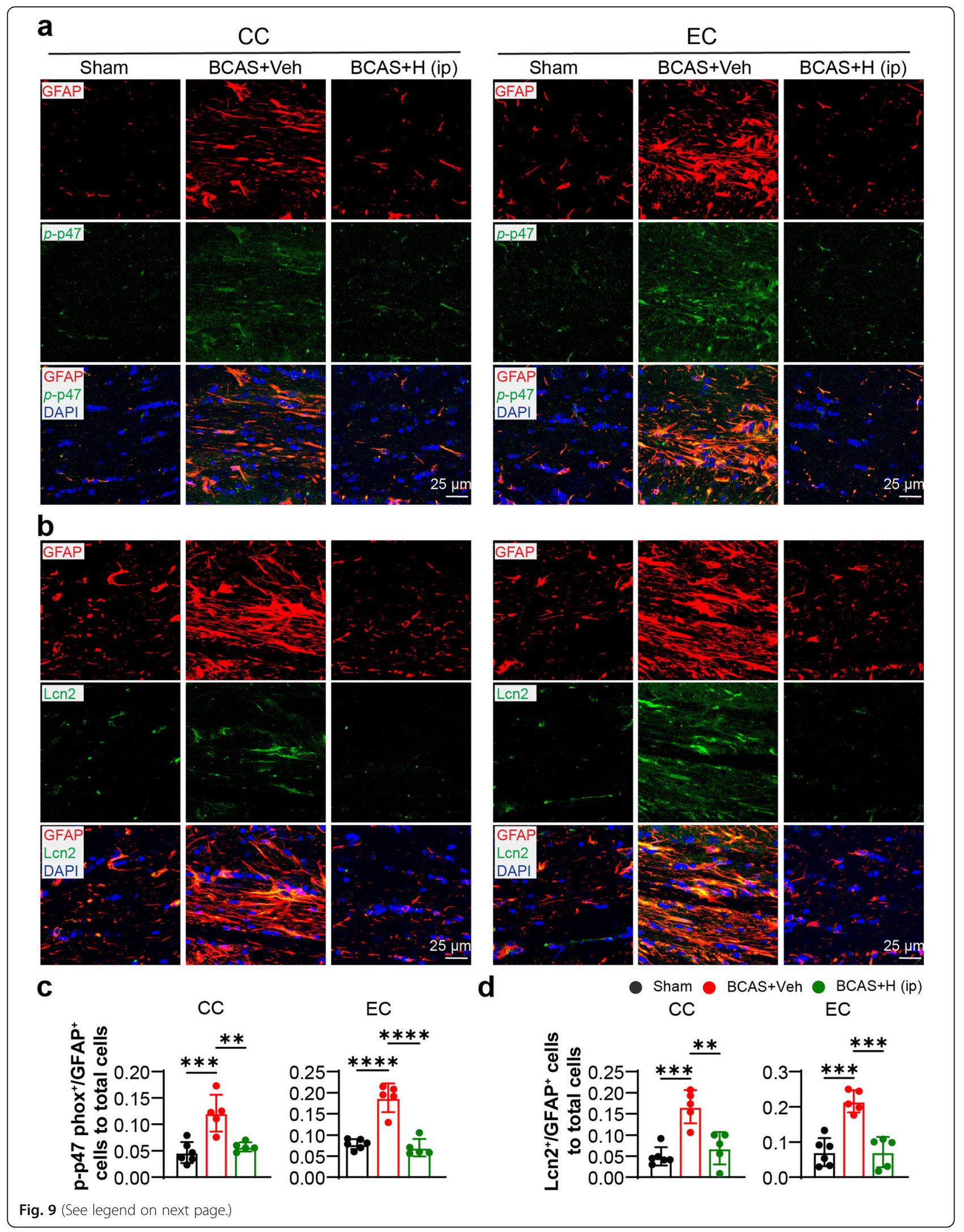


(See figure on previous page.)

Fig. 9 HOE642 treatment attenuated astrocytic NOX2 activation and LCN2 expression in white matter. a Representative staining images of phosphorylated p47 phox (green) and GFAP (red) in CC and EC at 30 days post-surgery. Magnification $\times 40$, Scale bar $=25 \mu \mathrm{m}$. b Representative immunostaining images of LCN2 (green) and GFAP (red) in CC and EC. Magnification ×40, scale bar $=25 \mu \mathrm{m}$. c Quantitative analysis of the cOlabeled p-p47 $/$ GFAP $^{+}$cells in CC and EC. $\mathbf{d}$ Quantitative analysis of the co-labeled LCN2 ${ }^{+} / \mathrm{GFAP}^{+}$cells in CC and EC. All data are presented as mean \pm SD. $n=5-6 .{ }^{* *} p<0.01,{ }^{* * *} p<0.001,{ }^{* * *} p<0.0001$

potential of targeting the astrocytic NHE1 protein for reducing astrogliosis in VCID.

\section{Correlation of brain microstructural damage, astroglial activation, and cognitive function impairment}

The strength of our study is that we completed cognitive behavioral tests, white matter microstructural analysis with MRI DTI, and evaluation of pathologic cellular changes with immunofluorescence staining in the same cohorts of mice, which allowed us to perform correlation analyses. Decreased FA is highly sensitive to detect white matter microstructural damage, and increased RD and decreased $\mathrm{AD}$ values are indicative of demyelination and axonal degeneration, respectively [34, 35]. We observed a linear correlation between the number of $\mathrm{GFAP}^{+}$reactive astrocytes and FA values in white matter (CC and $\mathrm{EC})$, which is consistent with reports for a detrimental role of astrogliosis in white matter lesions [32, 45]. However, in addition to MRI DTI and immunostaining analysis to assess the correlation of changes in MBP and astrocytic GFAP protein in white matter tissues, future Western blot quantification could further strengthen our findings. In addition, the FA values in white matter linearly correlated with the alternation performance in the $\mathrm{Y}$ maze test in mice, which suggests chronic hypoperfusion injury-mediated astrogliosis contributes to white matter lesions and ensuing spatial working memory deficit. We also detected a significant correlation between the number of reactive astrocytes in the hippocampus and the alternation performance in the $\mathrm{Y}$ maze test. Two treatment regimens with the selective NHE1 inhibitor HOE642 (cariporide) (daily bolus i.p. injection or continuous pump delivery for over 28 days) improved spatial working memory, likely due to inhibition of reactive astrogliosis in white matter and hippocampus. Blood-brain barrier (BBB) damage was detected as early as 1 day post BCAS surgery in mice [3, 46], which can facilitate HOE642 penetration into the BCAS parenchymal brain tissues. It is also reported that intravenous administration of HOE642 in rats is neuroprotective in permanent ischemic stroke model [47], another evidence for HOE642 penetration in ischemic brains with the compromised BBB.

However, some discrepancy in the correlative analysis of hippocampal astrogliosis and demyelination was detected in the HOE642 treatment (i.p.) mice, in which no improved hippocampal DTI indices (i.e. increased FA or decreased $\mathrm{RD}$ ) were observed. The reasons for this uncoupling are not apparent. Studies have reported that increased reactive astrogliosis could also elevate FA and decrease RD in DTI metrics due to their coherent arrangement or decreased the inter-space between axons resulting from axon swelling and astrocytic hypertrophy, which mask demyelination $[35,48]$. The lack of changes in the DTI metric values in the hippocampus of the BCAS+HOE (i.p.) mice could be due to the removal of the confounding influences of astrogliosis.

\section{Pharmacological inhibition of NHE1 protein reduces astrocytic proinflammatory transcriptome changes and NOX2 complex activation}

The underlying mechanisms for NHE1 in glial activation and inflammation are not completely understood. Our RNA-seq analysis of isolated astrocytes revealed that inhibition of NHE1 with HOE642 treatment substantially downregulated gene expressions involved cell cycle and inflammation responses to the BCAS-induced cerebral hypoperfusion. NHE1 blockade inhibited the gene expression of NOX2 (Cybb) and its subunit rac1/2 activityrelated genes (Racgap1, Rhoj, and Rnd1), which are involved in the production of ROS [49]. Moreover, in astrocytes of the BCAS+Veh mice, Prkch gene encoding protein kinase $\mathrm{C}$ (PKC), a key activator of NOX2 by phosphorylation of NOX2 subunits [39], was significantly upregulated, but the gene of PKC inhibitor (Ppp1r14a) was significantly downregulated. Interestingly, HOE642 treatment blocked the Prkch upregulation and Ppp1r14a downregulation, which could synergistically inhibit the phosphorylation of NOX2 subunits (p40, p47, p67). Our immunostaining data also showed that NHE1 inhibition attenuated NOX2 activity (reflected with reduced phosphorylation of the $\mathrm{p} 47$ subunit). In addition, the expression of the COX2 gene (Ptgst2), another important source of ROS [50] and canonical target of anti-chronic inflammation [51], was significantly reduced after the NHE1 inhibition. Inflammatory related gene product such as astrocytic LCN2 ( Lcn2) plays a pivotal role in gliosis, recruitment of macrophages, and release of pro-inflammatory cytokines [40]. Blocking NHE1 activity significantly attenuated LCN2 protein and mRNA expression in astrocytes.

The molecular underlying mechanisms are not known. We have previously reported that NHE1 proteinmediated $\mathrm{H}^{+}$extrusion in microglial cells prevents 
intracellular acidosis and is coupled with microglial activation and pro-inflammatory cytokine release via sustaining NOX2 function $[19,21]$. It has also been reported that NHE inhibitors (amiloride and EIPA) have antiinflammatory effects by decreasing LPS-induced COX2 expression and production of PGE2 in the murine macrophage cells [51], which may result from the alkalinization of extracellular environment [52]. Future studies are needed to determine whether NHE1-mediated regulation of $\mathrm{pH}_{\mathrm{i}}$ homeostasis in astrocytes directly modulates transcriptomes of ROS production and inflammation, and has an impact on ROS and cytokine production.

Pathological glial activation has a destructive effect on white matter integrity through their pro-inflammatory effects $[53,54]$. Reactive astrocytes showed significantly increased pro-inflammatory transcription factor $N F-k B$ activation and transgenic inhibition of astroglial NF- $\mathrm{kB}$ signaling preserved white matter integrity and improved cognitive function in BCAS mice [55]. Reactive astrocytes lost the ability to facilitate oligodendrocyte lineage cell maturation or developed abnormal glutamate metabolism [32, 56]. Moreover, there is close crosstalk between astrocytes and microglia in response to pathological stimuli [57]. Considering that the detrimental ROS and nitric oxide (NO) from reactive astrocytes can in turn to promote the activation of microglia [57, 58 ], the effects of pharmacological blockade of NHE1 protein in attenuating microglial activation could be indirect through reducing reactive astrogliosis and/or via directly inhibiting microglial NHE1 activity, the latter was shown to be involved in stroke-mediated proinflammatory microglial polarization [17].

A1, A2, and pan-reactive astrocyte transcriptomic signature analysis has been widely reported [37, 59]. We detected upregulation of genes in BCAS+Veh astrocytes, such as Lcn2, Serpina3n, Osmr, C3, C4b, which are wellknown pro-inflammatory markers of reactive astrocytes $[36,37]$. These cells did not show changes in top A2 phenotype genes $S 100 A 10$ or Ptx3 but displayed elevation of other A2 signature genes including Cd14, Ptgs 2 that are involved in inflammatory responses. Importantly, our results demonstrated that BCAS triggered reactive astrocyte response with upregulation of a range of inflammationrelated genes (Lcn2, Serpina3n, Osmr, C3, C4b, Cd14, Ptgs2, etc.), while NHE1 inhibitor HOE642 treatment suppressed these gene transcripts. These analyses indicate that the BCAS triggered distinctive phenotypes of reactive astrocyte transformation, which does not fall in the binary A1/A2 polarization, as reported recently [60].

\section{Protective effects of administration of NHE1 inhibitors in vivo}

Protective effects of pharmacological NHE1 inhibition have been reported in various brain injury studies. In mouse neonatal hypoxia-ischemia brain injury models, administration of NHE1 inhibitor HOE $642(0.5 \mathrm{mg} / \mathrm{kg}$, i.p.) in neonatal mice at $10 \mathrm{~min}, 24 \mathrm{~h}$, and $48 \mathrm{~h}$ after hypoxia reduced $\mathrm{CC}$ damage as well as hippocampal neurodegeneration $[23,61]$. In the mouse model of ischemic stroke, HOE $642(0.5 \mathrm{mg} / \mathrm{kg} / \mathrm{d}$, i.p. $)$ given at $0-7$ days post-stroke significantly decreased infarct volume, microglial activation, and pro-inflammatory stimulation, but did not attenuate reactive astrocytes in the periinfarct area [19]. Moreover, in a mouse global cerebral ischemia model by bilateral common carotid artery occlusion, administration of another NHE1 inhibitor amiloride $(10 \mathrm{mg} / \mathrm{kg}$, i.p.) after ischemia induction, significantly reduced neuronal loss, gliosis, and oxidative damage in the hippocampus [62]. Lastly, in a rat spontaneous subarachnoid hemorrhage model, intravenous injection of HOE642 $(15 \mathrm{mg} / \mathrm{kg})$ at $20 \mathrm{~min}$ before induction of subarachnoid hemorrhage decreased inflammatory reactions, oxidative stress, and neuronal loss [63]. In all these prior studies, NHE1 inhibitors were administered in the acute post-injury phase, which lasted no longer than one week. In contrast, in our study, HOE642 $(0.3 \mathrm{mk} / \mathrm{kg} /$ day $)$, administered for nearly 30 days, exhibited protective effects with improved cognitive functions and reduced brain pathology. These are encouraging new findings about the therapeutic potential for chronic NHE1 inhibition for the CCH-mediated brain damage.

However, the osmotic pump administration of HOE642 in our study was less effective with the underlying mechanisms still unknown. It could be due to HOE642 instability in the saline solution [64] in the osmotic pump or cumulative toxicity resulting from higher HOE642 plasma concentrations. Studies in a cardiac disease model have reported that the chronic administration of HOE642 (30mg/ $\mathrm{kg}$ /day) in rat drinking water for 1 month [65] or at 6000 ppm in mouse chow for 8 months [66] produced cardiac protective effects, without reported adverse effects. Considering the half-life of HOE642 at $~ 3.5 \mathrm{~h}$ in human serum and $1.5 \mathrm{~h}$ in rat serum $[67,68]$, there is a possibility that the osmotic pump with a constant delivery of HOE642 could accumulate higher serum concentrations and result in toxicity as indicated in the study by Kisker et al, that more than 5-fold increase in the area under the concentration time curve was detected in the osmotic pump delivery than in i.p. injection [69]. Future studies are needed to investigate the pharmacokinetics of HOE642 via different administration routes to determine the optimal dosing regimen. Additionally, the sample sizes in our study are relatively small; despite that, we were able to determine the statistical significance for neurological deficit assessment, immunostaining, and qRT-PCR and RNA-seq analyses. Determination of the potential therapeutic benefits of NHE1 pharmacological inhibition in the $\mathrm{CCH}$ model is warranted with larger sample sizes. 


\section{Conclusions}

Our study demonstrated that gliosis in white matter tracts and hippocampus is associated with white matter demyelination, microstructural damage of hippocampus, and spatial working memory deficits after chronic cerebral hypoperfusion injury induced by BCAS. Reactive $\mathrm{GFAP}^{+}$astrocytes displayed an elevated transcriptome for ROS production, inflammation, and NOX2 activation. Our study revealed that inhibition of NHE1 protein reduces brain astrocytic transcriptomes for ROS production and inflammation, as shown in the schematic summary in Supplemental Figure S10. Pharmacological inhibition of NHE1 activity with HOE642 attenuated reactive astrocyte gliosis, preserved white matter and hippocampal integrity, and improved cognitive function. These new findings suggest NHE1 protein as a potential therapeutic target for astrogliosis and VCID.

\begin{abstract}
Abbreviations
$\mathrm{CCH}$ : Chronic cerebral hypoperfusion; $\mathrm{NHE1}: \mathrm{Na}^{+} / \mathrm{H}^{+}$exchanger 1 ; BCAS: Bilateral carotid artery stenosis; MRI: Magnetic resonance imaging; DTI: Diffusion tensor imaging; DEC: Directionally encoded color; T2WI: T2weighted images; I.P. injection: Intraperitoneal injection; MBP: Myelin basic protein; NF-200: Neurofilament-200; GFAP: Glial fibrillary acidic protein; NADP $\mathrm{H}$ : Nicotinamide adenine dinucleotide phosphate; VCID: Vascular contributions to cognitive impairment and dementia; AD: Alzheimer's disease; WMLs: White matter lesions; NOX: NADPH oxidase; CBF: Cerebral blood flow; rCBF: Regional CBF; OF: Open field; CC: Corpus callosum; EC: External capsule; FA: Fractional anisotropy; AD: Axonal diffusivity; RD: Radial diffusivity; MD: Mean diffusivity; ROls: Regions of interest; DEGs: Differentially expressed genes; IPA: Ingenuity pathway analysis; GO: Gene ontology; DAVID: Database for Annotation Visualization and Integrated Discovery; SD: Standard deviation; SR: Stratum radiatum; PL: Polymorphic layer; SLM: Stratum lacunosum moleculare; ML: Molecular layer; ROS: Reactive oxygen species; COX2: Cyclooxygenase-2; Lcn2: Lipocalin 2; Rt-qPCR: Reverse transcription quantitative real-time PCR; PKC: Protein kinase $C$
\end{abstract}

\section{Supplementary Information}

The online version contains supplementary material available at https://doi. org/10.1186/s12974-021-02234-8.

\section{Additional file 1.}

\section{Acknowledgements}

CLC Genomics Workbench and Ingenuity Pathway Analysis software licensed through the Molecular Biology Information Service of the Health Sciences Library System, University of Pittsburgh, were used for data analysis.

\section{Authors' contributions}

Study conception and design: D. S., L. H., Q. L.; Experiment or data collection: Q. L., R.J. L., M.I. H B., S. S., G. B., C.B. Y., L. M. F., T.K. H.; data analysis: Q. L., R.J. L., S. S., M.I. H B., G. B., L. M. F., T.K. H.; data interpretation: D. S., Q. L., G.D. C.; writing-manuscript preparation and intellectual input: Q. L., D. S., L. H., G. B., M.I. H B., G.D. C., F.H., C.; supervision and administration: D. S. and L. H. The authors read and approved the final manuscript.

\section{Funding}

This research was supported in part by the University of Pittsburgh Center for Research Computing through the resources provided, UPMC Endowed Chair professorship for Brain Disorders Research (D.S), NIH R01NS048216 (D.S), VA Research Career Scientist award IK6 BX005647-01 (D.S), NIH RF1NS117509 (G.C) and VA Merit Review Grants BX003923 (G.C) and
BX002346 (G.C). Q. L. was in part supported by the China Scholarship Council 201906240207.

\section{Availability of data and materials}

Supporting data and information about used material are available from the corresponding author on reasonable request.

\section{Declarations}

Ethics approval and consent to participate

All animal experiments were conducted at university of Pittsburgh and were approved by the University of Pittsburgh Medical Center Institutional Animal Care and Use Committee (IACUC), which adhere to the National Institutes of Health Guide for the Care and Use of Laboratory Animals.

\section{Consent for publication}

Not applicable.

\section{Competing interests}

The authors declare that they have no competing interests.

\section{Author details}

${ }^{1}$ Department of Neurology, West China Hospital, Sichuan University, Chengdu 610041, Sichuan, China. ${ }^{2}$ Department of Neurology, University of Pittsburgh, Pittsburgh, Pennsylvania 15213, USA. ${ }^{3}$ Pittsburgh Institute for Neurodegenerative Disorders, University of Pittsburgh, Pittsburgh, Pennsylvania 15213, USA. ${ }^{4}$ Animal Imaging Center, University of Pittsburgh, Pittsburgh, Pennsylvania 15213, USA. 5 Department of Neurobiology, University of Pittsburgh, Pittsburgh, Pennsylvania 15213, USA. ${ }^{6}$ VA Pittsburgh Healthcare System, Geriatric Research Education and Clinical Center, Pittsburgh, Pennsylvania 15240, USA. ${ }^{7}$ Molecular Biology-Information Service, Health Sciences Library System, University of Pittsburgh, Pittsburgh, Pennsylvania 15261, USA.

Received: 17 June 2021 Accepted: 4 August 2021

Published online: 28 August 2021

References

1. Rizzi L, Rosset I, Roriz-Cruz M. Global epidemiology of dementia: Alzheimer's and vascular types. Biomed Res Int. 2014;2014:9089152014/08/05. https:// doi.org/10.1155/2014/908915.

2. Gladman JT, Corriveau RA, Debette $S$, et al. Vascular contributions to cognitive impairment and dementia: research consortia that focus on etiology and treatable targets to lessen the burden of dementia worldwide. Alzheimers Dement (N Y). 2019;5:789-962020/01/11. https://doi.org/10.1016/ j.trci.2019.09.017.

3. Duncombe J, Kitamura A, Hase Y, et al. Chronic cerebral hypoperfusion: a key mechanism leading to vascular cognitive impairment and dementia. Closing the translational gap between rodent models and human vascular cognitive impairment and dementia. Clin Sci (Lond). 2017, 2017/10/01;131: 2451-68. https://doi.org/10.1042/CS20160727.

4. Suri S, Topiwala A, Chappell MA, et al. Association of Midlife Cardiovascular Risk Profiles with Cerebral Perfusion at older ages. JAMA Netw Open. 2019;2: e1957762019/06/22. https://doi.org/10.1001/jamanetworkopen.2019.5776.

5. Staffaroni AM, Cobigo Y, Elahi FM, et al. Hum Brain Mapp. 2019;40:3522332019/05/08. https://doi.org/10.1002/hbm.24613.

6. Scherr M, Trinka E, Mc Coy M, et al. Cerebral hypoperfusion during carotid artery stenosis can lead to cognitive deficits that may be independent of white matter lesion load. Curr Neurovasc Res 2012; 9: 193-199. 2012/05/25. DOI: https://doi.org/10.2174/156720212801619009.

7. Romero JR, Beiser A, Seshadri S, et al. Carotid artery atherosclerosis, MR indices of brain ischemia, aging, and cognitive impairment: the Framingham study. Stroke 2009; 40: 1590-1596. 2009/03/07. DOl: https://doi. org/10.1161/STROKEAHA.108.535245.

8. Rosenberg GA. Extracellular matrix inflammation in vascular cognitive impairment and dementia. Clin Sci (Lond). 2017;131:425-37. 2017/03/08. https://doi.org/10.1042/CS20160604.

9. Chitnis T, Weiner HL, et al. J Clin Invest. 2017;127:3577-872017/09/06. https://doi.org/10.1172/JC190609.

10. Tangestani Fard M, Stough C. A review and hypothesized model of the mechanisms that underpin the relationship between inflammation and 
cognition in the elderly. Front Aging Neurosci. 2019;11:562019/04/02. https://doi.org/10.3389/fnagi.2019.00056.

11. Nordengen K, Kirsebom BE, Henjum K, et al. Glial activation and inflammation along the Alzheimer's disease continuum. J Neuroinflammation. 2019;16:462019/02/23. https://doi.org/10.1186/s12974019-1399-2.

12. Edison $P$, Donat CK and Sastre M. In vivo imaging of glial activation in Alzheimer's disease. Front Neurol 2018;9:625. 2018/08/23. https://doi.org/1 0.3389/fneur.2018.00625.

13. Low A, Mak E, Rowe JB, et al. Inflammation and cerebral small vessel disease: a systematic review. Ageing Res Rev. 2019;53:1009162019/06/11. https://doi.org/10.1016/j.arr.2019.100916.

14. Bowman GL, Dayon L, Kirkland R, Wojcik J, Peyratout G, Severin IC, et al. Blood-brain barrier breakdown, neuroinflammation, and cognitive decline in older adults. Alzheimers Dement. 2018;14:1640-50. 2018/08/19. https://doi. org/10.1016/j.jalz.2018.06.2857.

15. Chen Y, Tian $\mathrm{H}$, Yao E, et al. Soluble epoxide hydrolase inhibition promotes white matter integrity and long-term functional recovery after chronic hypoperfusion in mice. Sci Rep. 2017;7:77582017/08/12. https://doi.org/10.1 038/s41598-017-08227-z.

16. Begum $\mathrm{G}$, Song $\mathrm{S}$, Wang $\mathrm{S}$, et al. Selective knockout of astrocytic $\mathrm{Na}(+) / \mathrm{H}(+$ ) exchanger isoform 1 reduces astrogliosis, BBB damage, infarction, and improves neurological function after ischemic stroke. Glia. 2018;66:126442017/09/20. https://doi.org/10.1002/glia.23232.

17. Song $S$, Wang $S$, Pigott $V M$, et al. Selective role of $\mathrm{Na}(+) / H(+)$ exchanger in Cx3cr1 $1+$ ) microglial activation, white matter demyelination, and post-stroke function recovery. Glia. 2018;66:2279-98. 2018/07/26. https://doi.org/10.1 002/glia.23456.

18. Song $S$, Luo L, Sun $B$, et al. Roles of glial ion transporters in brain diseases. Glia. 2020;68:472-942019/08/17. https://doi.org/10.1002/glia.23699.

19. Shi $\mathrm{Y}$, Chanana V, Watters JJ, et al. Role of sodium/hydrogen exchanger isoform 1 in microglial activation and proinflammatory responses in ischemic brains. J Neurochem. 2011, 2011/07/30;119:124-35. https://doi. org/10.1111/j.1471-4159.2011.07403.x.

20. Cengiz $P$, Kintner DB, Chanana V, et al. Sustained $\mathrm{Na}+/ \mathrm{H}+$ exchanger activation promotes gliotransmitter release from reactive hippocampal astrocytes following oxygen-glucose deprivation. PLoS One. 2014;9:e84294. 2014/01/07. https://doi.org/10.1371/journal.pone.0084294.

21. Lam TI, Brennan-Minnella AM, Won SJ, et al. Intracellular pH reduction prevents excitotoxic and ischemic neuronal death by inhibiting NADPH oxidase. Proc Natl Acad Sci U S A. 2013;110:E4362-8. 2013/10/29. https://doi. org/10.1073/pnas.1313029110.

22. Bondarenko A, Svichar N, Chesler M. Role of Na+-H+ and $\mathrm{Na}+-\mathrm{Ca} 2+$ exchange in hypoxia-related acute astrocyte death. Glia. 2005;49:143522004/09/25. https://doi.org/10.1002/glia.20107.

23. Cengiz $\mathrm{P}$, Uluc K, Kendigelen $\mathrm{P}$, et al. Chronic neurological deficits in mice after perinatal hypoxia and ischemia correlate with hemispheric tissue loss and white matter injury detected by MRI. Dev Neurosci. 2011;33:270-92011/ 06/28. https://doi.org/10.1159/000328430.

24. Percie du Sert N, Hurst V, Ahluwalia A, et al. The ARRIVE guidelines 2.0: updated guidelines for reporting animal research. J Cereb Blood Flow Metab. 2020;40:1769-77. 2020/07/15. https://doi.org/10.1177/0271678X2 0943823.

25. Masamoto K, Fukuda M, Vazquez A, et al. Dose-dependent effect of isoflurane on neurovascular coupling in rat cerebral cortex. Eur J Neurosci. 2009;30:24250. 2009/08/08. https://doi.org/10.1111/j.1460-9568.2009.06812.x.

26. Munting LP, Derieppe MPP, Suidgeest $E$, et al. Influence of different isoflurane anesthesia protocols on murine cerebral hemodynamics measured with pseudo-continuous arterial spin labeling. NMR Biomed. 2019;32:e4105. 2019/06/07. https://doi.org/10.1002/nbm.4105.

27. Sukoff Rizzo SJ, Anderson LC, Green TL, et al. Assessing Healthspan and lifespan measures in aging mice: optimization of testing protocols, replicability, and rater reliability. Curr Protoc Mouse Biol. 2018;8:e45. 2018/ 06/21. https://doi.org/10.1002/cpmo.45.

28. Zhang J, Bhuiyan MIH, Zhang T, et al. Modulation of brain cation-cl(-) cotransport via the SPAK kinase inhibitor ZT-1a. Nat Commun. 2020;11:78. 2020/01/09. https://doi.org/10.1038/s41467-019-13851-6.

29. Song $\mathrm{S}$, Huang $\mathrm{H}$, Guan $\mathrm{X}$, et al. Activation of endothelial Wnt/beta-catenin signaling by protective astrocytes repairs BBB damage in ischemic stroke. Prog Neurobiol. 2020:101963. 2020/11/30. https://doi.org/10.1016/j. pneurobio.2020.101963.
30. Kramer A, Green J, Pollard J Jr, et al. Causal analysis approaches in ingenuity pathway analysis. Bioinformatics. 2014;30:523-30. 2013/12/18. https://doi. org/10.1093/bioinformatics/btt703.

31. Huang DW, Sherman BT, Lempicki RA. Systematic and integrative analysis of large gene lists using DAVID bioinformatics resources. Nat Protoc. 2009;4: 44-57. https://doi.org/10.1038/nprot.2008.211.

32. Miyamoto N, Magami S, Inaba T, et al. The effects of A1/A2 astrocytes on oligodendrocyte linage cells against white matter injury under prolonged cerebral hypoperfusion. Glia. 2020 2020/02/29. https://doi.org/10.1002/ glia.23814.

33. Al-Onaizi MA, Parfitt GM, Kolisnyk B, et al. Regulation of cognitive processing by hippocampal cholinergic tone. Cereb Cortex. 2017;27:1615-28. 2016/01/ 24. https://doi.org/10.1093/cercor/bhv349.

34. Alexander $\mathrm{AL}$, Lee JE, Lazar $\mathrm{M}$, et al. Diffusion tensor imaging of the brain. Neurotherapeutics. 2007:4:316-29. 2007/06/30. https://doi.org/10.1016/j. nurt.2007.05.011.

35. Budde MD, Janes L, Gold E, et al. The contribution of gliosis to diffusion tensor anisotropy and tractography following traumatic brain injury: validation in the rat using Fourier analysis of stained tissue sections. Brain. 2011;134:2248-60. 2011/07/19. https://doi.org/10.1093/brain/awr161.

36. Das S, Li Z, Noori A, et al. Meta-analysis of mouse transcriptomic studies supports a context-dependent astrocyte reaction in acute CNS injury versus neurodegeneration. J Neuroinflammation. 2020;17:227. 2020/08/02. https:// doi.org/10.1186/s12974-020-01898-y.

37. Liddelow SA, Guttenplan KA, Clarke LE, et al. Neurotoxic reactive astrocytes are induced by activated microglia. Nature. 2017;541:481-7. 2017/01/19. https://doi.org/10.1038/nature21029.

38. Liu Y, Kintner DB, Chanana V, et al. Activation of microglia depends on $\mathrm{Na}+$ / $\mathrm{H}+$ exchange-mediated $\mathrm{H}+$ homeostasis. J Neurosci. 2010;30:15210-20. 2010/11/12. https://doi.org/10.1523/JNEUROSCl.3950-10.2010.

39. Raad H, Paclet MH, Boussetta T, et al. Regulation of the phagocyte NADPH oxidase activity: phosphorylation of gp91 phox/NOX2 by protein kinase $C$ enhances its diaphorase activity and binding to Rac2, p67phox, and p47phox. FASEB J. 2009;23:1011-22. 2008/11/26. https://doi.org/10.1096/fj. 08-114553.

40. Suk K. Lipocalin-2 as a therapeutic target for brain injury: an astrocentric perspective. Prog Neurobiol. 2016;144:158-72. 2016/08/09. https://doi.org/1 0.1016/j.pneurobio.2016.08.001.

41. Roman GC. Brain hypoperfusion: a critical factor in vascular dementia. Neurol Res. 2004;26:454-8. 2004/07/22. https://doi.org/10.1179/01616410422 5017686.

42. Caroli A, Testa C, Geroldi C, et al. Brain perfusion correlates of medial temporal lobe atrophy and white matter hyperintensities in mild cognitive impairment. J Neurol. 2007;254:1000-8. 2007/03/22. https://doi.org/10.1007/ s00415-006-0498-z.

43. Inaba T, Miyamoto N, Hira K, et al. Protective role of Levetiracetam against cognitive impairment and brain white matter damage in mouse prolonged cerebral Hypoperfusion. Neuroscience. 2019;414:255-64. 2019/07/16. https:// doi.org/10.1016/j.neuroscience.2019.07.015.

44. Roberts JM, Maniskas ME, Bix GJ. Bilateral carotid artery stenosis causes unexpected early changes in brain extracellular matrix and blood-brain barrier integrity in mice. PLoS One. 2018;13:e0195765. 2018/04/13. https:// doi.org/10.1371/journal.pone.0195765.

45. Kuipers HF, Yoon J, van Horssen J, et al. Phosphorylation of alphaB-crystallin supports reactive astrogliosis in demyelination. Proc Natl Acad Sci U S A. 2017: 114, E1745-E1754. 2017/02/16. https://doi.org/10.1073/pnas.1621314114.

46. Liu Q, Radwanski R, Babadjouni R, et al. Experimental chronic cerebral hypoperfusion results in decreased pericyte coverage and increased bloodbrain barrier permeability in the corpus callosum. J Cereb Blood Flow Metab. 2019;39:240-50. 2017/12/02. https://doi.org/10.1177/0271678X17743670.

47. O'Donnell ME, Chen YJ, Lam TI, et al. Intravenous HOE-642 reduces brain edema and $\mathrm{Na}$ uptake in the rat permanent middle cerebral artery occlusion model of stroke: evidence for participation of the blood-brain barrier Na/H exchanger. J Cereb Blood Flow Metab. 2013;33:225-34. 2012/ 11/15. https://doi.org/10.1038/jcbfm.2012.160.

48. Stephen R, Solomon A, Ngandu T, et al. White matter changes on diffusion tensor imaging in the FINGER randomized controlled trial. J Alzheimers Dis. 2020;78:75-86. 2020/09/15. https://doi.org/10.3233/JAD-200423.

49. Hordijk PL. Regulation of NADPH oxidases: the role of Rac proteins. Circ Res. 2006;98:453-62. 2006/03/04. https://doi.org/10.1161/01.RES.0000204727.4671 $0.5 \mathrm{e}$. 
50. Munoz M, Sanchez A, Pilar Martinez M, et al. COX-2 is involved in vascular oxidative stress and endothelial dysfunction of renal interlobar arteries from obese Zucker rats. Free Radic Biol Med. 2015;84:77-90. 2015/04/07. https:// doi.org/10.1016/j.freeradbiomed.2015.03.024

51. Kamachi F, Ban HS, Hirasawa N, et al. Inhibition of lipopolysaccharideinduced prostaglandin E2 production and inflammation by the $\mathrm{Na}+\mathrm{H}+$ exchanger inhibitors. J Pharmacol Exp Ther. 2007;321:345-52. 2007/01/24. https://doi.org/10.1124/jpet.106.116251.

52. Tomura H, Wang JQ, Liu JP, et al. Cyclooxygenase-2 expression and prostaglandin E2 production in response to acidic $\mathrm{pH}$ through OGR1 in a human osteoblastic cell line. J Bone Miner Res. 2008;23:1129-39. 2008/02/ 28. https://doi.org/10.1359/jbmr.080236.

53. Miyanohara J, Kakae M, Nagayasu K, et al. TRPM2 channel aggravates CNS inflammation and cognitive impairment via activation of microglia in chronic cerebral Hypoperfusion. J Neurosci. 2018;38:3520-33. 2018/03/07. https://doi.org/10.1523/JNEUROSCI.2451-17.2018.

54. Hou X, Liang X, Chen JF, et al. Ecto-5'-nucleotidase (CD73) is involved in chronic cerebral hypoperfusion-induced white matter lesions and cognitive impairment by regulating glial cell activation and pro-inflammatory cytokines. Neuroscience. 2015;297:118-26. 2015/03/26. https://doi.org/10.101 6/j.neuroscience.2015.03.033.

55. Saggu R, Schumacher T, Gerich F, et al. Astroglial NF-kB contributes to white matter damage and cognitive impairment in a mouse model of vascular dementia. Acta Neuropathol Commun. 2016;4:76. 2016/08/05. https://doi. org/10.1186/s40478-016-0350-3.

56. Vicente E, Degerone D, Bohn L, et al. Astroglial and cognitive effects of chronic cerebral hypoperfusion in the rat. Brain Res. 2009;1251:204-12. 2008/12/06. https://doi.org/10.1016/j.brainres.2008.11.032.

57. Matejuk A, Ransohoff RM. Crosstalk between astrocytes and microglia: an overview. Front Immunol. 2020;11. Review. https://doi.org/10.3389/fimmu.2 020.01416.

58. Chun $\mathrm{H}, \mathrm{Im} H$, Kang $Y$ J, et al. Severe reactive astrocytes precipitate pathological hallmarks of Alzheimer's disease via H2O2(-) production. Nat Neurosci. 2020;23:1555-66. 2020/11/18. https://doi.org/10.1038/s41593-02000735-y.

59. Clarke LE, Liddelow SA, Chakraborty C, et al. Normal aging induces A1-like astrocyte reactivity. Proc Natl Acad Sci U S A. 2018;115:E1896-905. 2018/02/ 14. https://doi.org/10.1073/pnas.1800165115.

60. Escartin C, Galea E, Lakatos A, et al. Reactive astrocyte nomenclature, definitions, and future directions. Nat Neurosci. 2021;24:312-25. 2021/02/17. https://doi.org/10.1038/s41593-020-00783-4

61. Cengiz P, Kleman N, Uluc K, Kendigelen P, Hagemann T, Akture E, et al. Inhibition of $\mathrm{Na}+/ \mathrm{H}+$ exchanger isoform 1 is neuroprotective in neonatal hypoxic ischemic brain injury. Antioxid Redox Signal. 2011;14:1803-13. 2010/08/18. https://doi.org/10.1089/ars.2010.3468.

62. Kang BS, Choi BY, Kho AR, et al. An inhibitor of the sodium-hydrogen Exchanger-1 (NHE-1), Amiloride, reduced zinc accumulation and hippocampal neuronal death after ischemia. Int J Mol Sci. 2020; 21 2020/06/ 18. https://doi.org/10.3390/ijms21124232.

63. Song H, Yuan S, Zhang Z, et al. Sodium/hydrogen exchanger 1 participates in early brain injury after subarachnoid hemorrhage both in vivo and in vitro via promoting neuronal apoptosis. Cell Transplant. 2019;28:9851001. 2019/03/07. https://doi.org/10.1177/0963689719834873.

64. Baumgarth M, Beier N, Gericke R. (2-Methyl-5(methylsulfonyl)benzoyl)guanidine $\mathrm{Na}+/ \mathrm{H}+$ antiporter inhibitors. J Med Chem. 1997;40:2017-34. 1997/06/20. https://doi.org/10.1021/ jm960768n.

65. Garciarena CD, Caldiz Cl, Portiansky EL, et al. Chronic NHE-1 blockade induces an antiapoptotic effect in the hypertrophied heart. J Appl Physiol (1985). 2009;106:1325-31. 2009/01/31. https://doi.org/10.1152/japplphysiol. 91300.2008 .

66. Engelhardt $\mathrm{S}$, Hein $\mathrm{L}$, Keller $\mathrm{U}$, et al. Inhibition of $\mathrm{Na}(+)-\mathrm{H}(+)$ exchange prevents hypertrophy, fibrosis, and heart failure in beta(1)-adrenergic receptor transgenic mice. Circ Res. 2002;90:814-9. 2002/04/20. https://doi. org/10.1161/01.res.0000014966.97486.c0.

67. Spitznagel $\mathrm{H}$, Chung $\mathrm{O}$, Xia Q, et al. Cardioprotective effects of the $\mathrm{Na}(+$ )/H(+)-exchange inhibitor cariporide in infarct-induced heart failure. Cardiovasc Res. 2000;46:102-10. 2000/03/23. https://doi.org/10.1016/s00086363(99)00428-9

68. Guan X, Hasan MN, Begum G, et al. Blockade of $\mathrm{Na} / \mathrm{H}$ exchanger stimulates glioma tumor immunogenicity and enhances combinatorial TMZ and anti-
PD-1 therapy. Cell Death Dis. 2018;9:1010. 2018/09/29. https://doi.org/10.103 8/s41419-018-1062-3.

69. Kisker O, Becker CM, Prox D, et al. Continuous administration of endostatin by intraperitoneally implanted osmotic pump improves the efficacy and potency of therapy in a mouse xenograft tumor model. Cancer Res. 2001; 61:7669-74 2001/10/19.

\section{Publisher's Note}

Springer Nature remains neutral with regard to jurisdictional claims in published maps and institutional affiliations.
Ready to submit your research? Choose BMC and benefit from:

- fast, convenient online submission

- thorough peer review by experienced researchers in your field

- rapid publication on acceptance

- support for research data, including large and complex data types

- gold Open Access which fosters wider collaboration and increased citations

- maximum visibility for your research: over $100 \mathrm{M}$ website views per year

At BMC, research is always in progress.

Learn more biomedcentral.com/submissions 